\title{
ON THE BEHAVIOR OF TRIGONOMETRIC SERIES AND POWER SERIES
}

\author{
BY
}

\section{J. MARCINKIEWICZ AND A. ZYGMUND \\ INTRODUCTION}

1.1. The present paper consists of two parts. The first part contains proofs of theorems describing the behavior of the partial sums of trigonometric series. The second part gives corresponding results for the partial sums of power series on the circle of convergence.

Let us consider the trigonometric series

$$
\frac{1}{2} a_{0}+\sum_{\nu=1}^{\infty}\left(a_{\nu} \cos \nu \theta+b_{\nu} \sin \nu \theta\right)
$$

and the conjugate series

$$
\sum_{\nu=1}^{\infty}\left(a_{\nu} \sin \nu \theta-b_{\nu} \cos \nu \theta\right) .
$$

The partial sums of these two series will be denoted by $s_{n}(\theta)$ and $\tilde{s}_{n}(\theta)$ respectively. The Cesàro means of order $\alpha$ of the series (1.1.1) and (1.1.2) will be denoted by $\sigma_{n}^{\alpha}(\theta)$ and $\left.\tilde{\sigma}_{n}^{\alpha}(\theta){ }^{1}\right)$. We also introduce the following notation:

$$
\liminf _{n \rightarrow \infty} \sigma_{n}^{\alpha}(\theta)=\sigma_{\alpha}(\theta), \quad \quad \quad \limsup _{n \rightarrow \infty} \sigma_{n}^{\alpha}(\theta)=\sigma^{\alpha}(\theta), \quad \sigma^{\alpha}(\theta)-\sigma_{\alpha}(\theta)=\omega_{\alpha}(\theta) .
$$

The last expression represents the oscillation of the sequence $\left\{\sigma_{n}^{\alpha}(\theta)\right\}$ for $n \rightarrow+\infty$. If $\sigma_{n}^{\alpha}(\theta)$ tends to $+\infty$ or to $-\infty$, we write $\omega_{\alpha}(\theta)=+\infty$. Hence the condition $\omega_{\alpha}(\theta)=0$ is both necessary and sufficient for the series (1.1.1) to be summable $(C, \alpha)$ at the point $\theta$.

Similarly, we write

$$
\liminf _{n \rightarrow \infty} \tilde{\sigma}_{n}^{\alpha}(\theta)=\sigma_{\alpha}(\theta), \quad \quad \limsup _{n \rightarrow \infty} \tilde{\sigma}_{n}^{\alpha}(\theta)=\tilde{\sigma}^{\alpha}(\theta), \quad \tilde{\sigma}^{\alpha}(\theta)-\tilde{\sigma}_{\alpha}(\theta)=\tilde{\omega}_{\alpha}(\theta) .
$$

Theorems 1 and 2 which follow connect the behavior of the series (1.1.1) and (1.1.2). By summability $A$ we mean Abel summability.

Theorem 1. Suppose that the series (1.1.1) is summable $A$ to sum $s(\theta)$ at every point of a set $E$ of positive measure, and that for an $\alpha>-1$

Presented to the Society, January 1, 1941; received by the editors October 10, 1940.

(1) In $\$ 1.5$ below a few properties of the arithmetic means are collected which will be used in the sequel. 


$$
\sigma_{\alpha}(\theta)>-\infty
$$

Then at almost every point of $E$ we have the following relations:

$$
\begin{aligned}
\sigma^{\alpha}(\theta) & <+\infty, \\
-\infty<\tilde{\sigma}_{\alpha}(\theta) & \leqq \tilde{\sigma}^{\alpha}(\theta)<+\infty, \\
\tilde{\omega}_{\alpha}(\theta) & =\omega_{\alpha}(\theta) .
\end{aligned}
$$

Moreover the series (1.1.1) and (1.1.2) are summable $(C, \alpha+\epsilon), \epsilon>0$, at almost every point of the set $E$, and

$$
\begin{aligned}
& s(\theta)=\frac{1}{2}\left\{\sigma_{\alpha}(\theta)+\sigma^{\alpha}(\theta)\right\}, \\
& \tilde{s}(\theta)=\frac{1}{2}\left\{\tilde{\sigma}_{\alpha}(\theta)+\tilde{\sigma}^{\alpha}(\theta)\right\}
\end{aligned}
$$

almost everywhere in $E$, where $\tilde{s}(\theta)$ denotes the $(C, \alpha+\epsilon)$ sum of the series (1.1.2).

If the hypotheses of Theorem 1 are satisfied, we have therefore that the $\alpha$ th Cesàro means of the series (1.1.1) and (1.1.2) are bounded at almost every point of $E$.

From (1.1.6) we see that the oscillations of the sequences $\left\{\sigma_{n}^{\alpha}(\theta)\right\}$ and $\left\{\tilde{\sigma}_{n}^{\alpha}(\theta)\right\}$ are equal at almost every point of the set $E$. In particular, if one of the series (1.1.1) and (1.1.2) is summable $(C, \alpha)$ at almost every point of $E$, so is the other.

The relations (1.1.7) and (1.1.8) show that the limits of indetermination of the sequences $\left\{\sigma_{n}^{\alpha}(\theta)\right\}$ and $\left\{\tilde{\sigma}_{n}^{\alpha}(\theta)\right\}$ are situated symmetrically with respect to the (generalized) sums of the series (1.1.1) and (1.1.2).

It follows at once from Theorem 1 that, if the series (1.1.1) is summable $A$ at every point of the set $E$, and if $\sigma_{\alpha}(\theta)=-\infty$ in $E$, then $\sigma^{\alpha}(\theta)=+\infty$ almost everywhere in $E$. The question whether we also have

$$
\tilde{\sigma}_{\alpha}(\theta)=-\infty, \quad \tilde{\sigma}^{\alpha}(\theta)=+\infty
$$

almost everywhere in $E$ is left open.

Theorem 2. Suppose that the ath Cesàro means (where $\alpha>-1$ ) of the series (1.1.1) are bounded at every point of a set $E$ of positive measure, that is, that

$$
\sigma_{n}^{\alpha}(\theta)=O(1)
$$

for every $\theta \in E$.

Then at almost every point of $E$ the series (1.1.1) and (1.1.2) are summable $(C, \alpha+\epsilon)(\epsilon>0)$ and we have the relations (1.1.5), (1.1.6), (1.1.7) and (1.1.8).

The hypothesis (1.1.3) of one-sided boundedness, in Theorem 1, is replaced in Theorem 2 by the stronger hypothesis (1.1.9); but, on the other hand, no assumption is made in Theorem 2 concerning the summability of the series (1.1.1).

Theorems 1 and 2 are known. Special cases of Theorem 1 have been proved 
by Kuttner $\left({ }^{2}\right)$ and Plessner $\left({ }^{3}\right)$, and the general result stated above was proved by the authors $\left({ }^{4}\right)$.

The original proofs of Theorems 1 and 2 are however rather difficult. In the first part of this paper we give new proofs of these theorems, using certain ideas of Plessner, in particular the application of summability $\left(C^{*}, \alpha\right)$ (defined in $\$ 2.1$ below $\left.{ }^{5}\right)$ ). The chief simplification in comparison with the earlier proofs (in particular with Plessner's proof) is that we do not require the theory of higher generalized derivatives.

1.2. Theorems 1 and 2 raise the problem of the behavior of the Cesàro means of a power series on the circle of convergence. Let us begin first with the case of partial sums.

Let

$$
F(z)=\sum_{\nu=0}^{\infty} c_{\nu} z^{\nu}, \quad|z|<1,
$$

be a function regular inside the unit circle. We may suppose for the moment without loss of generality (subtracting a constant from $F(z)$ if necessary) that $c_{0}$ is real. If

$$
c_{n}=a_{n}-i b_{n} \quad \text { for } n=1,2,3, \cdots,
$$

then the real and imaginary parts of the series

$$
\sum_{\nu=0}^{\infty} c_{\nu} e^{i \nu \theta}
$$

may be written in the forms (1.1.1) and (1.1.2) respectively.

Let us assume that the partial sums of the series (1.2.1) are bounded at every point $z=e^{i \theta}$ of a certain set $E$ situated on the circumference $|z|=1$. We shall also denote by $E$ the set of the corresponding arguments $\theta$; this, however, will not lead to any confusion. By Theorem 2 the series (1.2.2) is summable $(C, 1)$ to sum $t(\theta)$ at almost every point of $E$. It follows from Theorem 1 , therefore, that for almost every point $\theta$ of $E$ the limit points of the sequence

$$
t_{n}(\theta)=\sum_{\nu=0}^{n} c_{\nu} e^{i \nu \theta}, \quad n=0,1,2, \cdots,
$$

(2) Kuttner [1] proves that if the series (1.1.1) converges in $E$ and the series (1.1.2) is summable $(C, 1)$ in $E$, then the latter series converges almost everywhere in $E$.

${ }^{(3)}$ The result of Plessner [1] coincides with Lemma $M$ of the present paper in the case $\beta=0,1,2, \cdots$. Plessner only sketches the proof of the result.

(4) See Marcinkiewicz and Zygmund [1].

(5) That notion was considered independently by one of the authors and applied to the theory of Fourier series. See Zygmund [1, p. 61, Example 4].

It must however be observed that the expressions

$$
\theta_{n}^{\alpha}\left(\theta+h_{n}\right) \pm \sigma_{n}^{\alpha}\left(\theta-h_{n}\right)
$$

which play an essential part in our argument have been studied for the first time by Rogosinski [1]. 
belong to the square with center $t(\theta)$ and sides $\omega_{0}(\theta)$, parallel to the axes. Here $\omega_{0}(\theta)$ denotes the oscillation of the real part of the series (1.2.2). By Theorem 2, this square cannot be replaced by a smaller rectangle with the same center and sides parallel to the axes. On the other hand, not every point of the square need be a limit point of the sequence $\left\{t_{n}(\theta)\right\}$, as simple examples show.

In fact, take the series

$$
1+z+z^{2}+\cdots
$$

the partial sums of which are bounded and summable $(C, 1)$ at every point $z$ of $|z|=1$ except $z=1$. Here

$$
t(\theta)=\frac{1}{1-e^{i \theta}}, \quad t_{n}(\theta)-t(\theta)=-\frac{e^{i(n+1) \theta}}{1-e^{i \theta}},
$$

so that all the partial sums of the series (1.2.3), with $z=e^{i \theta}, \theta \neq 0(\bmod 2 \pi)$, are situated on the circle with center $\left(1-e^{i \theta}\right)^{-1}$ and radius $\left|1-e^{i \theta}\right|^{-1}$. The same is of course true of the set of the limit points of the sequence $\left\{t_{n}(\theta)\right\}$. Moreover, if $\theta$ is not commensurable with $\pi$, every point of the circle just defined is a limit of the sequence $\left\{t_{n}(\theta)\right\}$.

Let us modify this example slightly by considering the power series

$$
\begin{aligned}
(\alpha+\beta)+\alpha z+(\alpha & +\beta) z^{2}+\alpha z^{3}+\cdots \\
& =\alpha\left(1+z+z^{2}+\cdots\right)+\beta\left(1+z^{2}+z^{4}+\cdots\right),
\end{aligned}
$$

where $\alpha$ and $\beta$ are any constants. It may be verified without difficulty that in this case, for $\theta$ different from 0 and $\pi$, the partial sums $t_{n}(\theta)$ are situated on two concentric circles, with center

and radii

$$
\frac{\alpha}{1-e^{i \theta}}+\frac{\beta}{1-e^{2 i \theta}}
$$

$$
\frac{1}{\left|1-e^{i \theta}\right|} \cdot\left|\alpha+\frac{\beta}{1+e^{ \pm i \theta}}\right| \text {. }
$$

These two circles are different if the ratio $\beta / \alpha$ is not real. If $\theta$ is incommensurable with $\pi$, every point of these two circles is a limit point of the sequence $\left\{t_{n}(\theta)\right\}$.

Similarly, in the case of the series

$(1.2 .5) \alpha\left(1+z+z^{2}+\cdots\right)+\beta\left(1+z^{2}+z^{4}+\cdots\right)+\gamma\left(1+z^{3}+z^{6}+\cdots\right.$

the partial sums $t_{n}(\theta)$ are situated on six (generally distinct) circles with center

$$
t(\theta)=\frac{\alpha}{1-e^{i \theta}}+\frac{\beta}{1-e^{2 i \theta}}+\frac{\gamma}{1-e^{3 i \theta}},
$$


and if $\theta$ is incommensurable with $\pi$, every point of each of those six circles is a limit point of the sequence $\left\{t_{n}(\theta)\right\}$.

A slightly different example may be obtained by considering the series

$$
\left(1+z+z^{2}+\cdots\right)+\alpha\left(1+e^{i \lambda} z+e^{2 i \lambda} z^{2}+\cdots\right), \quad z=e^{i \theta},
$$

where $a \neq 0$ and $\lambda$ is a real number incommensurable with $\pi$. If $\theta$ is linearly independent of $\lambda$, and $n$ is suitably chosen, the fractional parts of $(n+1) \theta$ and $(n+1) \lambda$ may be as close as we wish to any preassigned numbers of the interval $(0,1)$. Using this fact, we easily obtain that, except for a denumerable set of values of $\theta$, the limit points of the sequence of the partial sums of our series, with $z=e^{i \theta}$, form an annulus not reducing to a circle or a circumference.

By combining this example with the preceding ones, we may obtain a power series such that for values of $\theta$ belonging to a set of positive measure the set of limit points of the partial sums will consist of several concentric annuli.

The particular series which we have just considered illustrate some general theorems which will be stated in a moment. It will be convenient to introduce certain notations.

By

$$
\mathrm{A}\left\{z_{0} ; \alpha, \beta\right\}
$$

where $0 \leqq \alpha \leqq \beta \leqq \infty$, we shall mean the annulus consisting of the points $z$ satisfying the inequalities $\alpha \leqq\left|z-z_{0}\right| \leqq \beta$. The circle $\mathrm{A}\left\{z_{0} ; 0, \beta\right\}$ will be denoted simply by

$$
\mathrm{K}\left\{z_{0} ; \beta\right\},
$$

and the circumference $\left|z-z_{0}\right|=\beta$ by

$$
\mathrm{C}\left\{z_{0} ; \beta\right\} \text {. }
$$

We shall say that a plane set $Z$ is of circular structure, if there is a point $z_{0}$ (center of $Z$ ) such that whenever a point $\zeta$ belongs to $Z$ so does the whole circumference $\mathrm{C}\left\{z_{0} ;\left|\zeta-z_{0}\right|\right\}$.

Given a function $F(z)$ defined by (1.2.1) we shall write

$$
\begin{aligned}
& M(\theta)=M_{F}(\theta)=\limsup _{n \rightarrow \infty}\left|t_{n}(\theta)-t(\theta)\right|, \\
& m(\theta)=m_{F}(\theta)=\liminf _{n \rightarrow \infty}\left|t_{n}(\theta)-t(\theta)\right|,
\end{aligned}
$$

where $t(\theta)$ is the $(C, 1)$ sum (provided it exists) of the series (1.2.2). The set of the limit points of the sequence $\left\{t_{n}(\theta)\right\}$, when $\theta$ is fixed, will be denoted by $\mathrm{L}_{F}(\theta)$, or simply by $\mathrm{L}(\theta)$.

Now we may state the following

TheOREM A. Suppose that the partial sums of the series (1.2.1) are bounded at every point of a set $E$ situated on the circumference $\mathrm{C}\{0 ; 1\}$ and let $t(\theta)$ denote 
the $(C, 1)$ sum (existing, by Theorem 2, almost everywhere in $E$ ) of the series (1.2.2). Then, for almost every $\theta \in E$, the set $\mathrm{L}(\theta)$ is of circular structure, with center $t(\theta)$.

In other words, for almost every value of $\theta$ contained in $E$ the set of the limit points of the sequence $\left\{t_{n}(\theta)\right\}$ consists of a finite, denumerable, or nondenumerable set of circumferences with center at $t(\theta)$. The set $\mathrm{L}(\theta)$ is obviously contained in the annulus $\mathrm{A}\{t(\theta) ; m(\theta), M(\theta)\}$. The set $\mathrm{L}(\theta)$ being closed, the extreme circumferences $\mathrm{C}\{t(\theta) ; m(\theta)\}$ and $\mathrm{C}\{t(\theta) ; M(\theta)\}$ of that annulus belong to $\mathrm{L}(\theta)$.

If the partial sums of the series (1.2.2) are bounded for some $\theta=\theta_{0}$, the coefficients $c_{n}$ are bounded. The examples of the series (1.2.4) and (1.2.5) show that the set $\mathrm{L}(\theta)$ need not coincide with $\mathrm{A}\{t(\theta) ; m(\theta), M(\theta)\}$. This however will be the case, if we assume additionally that the coefficients $c_{n}$ tend to 0 .

In other words, we have the following theorem:

ThEOREM B. Suppose that the series (1.2.2) satisfies the conditions of Theorem $\mathrm{A}$ and that $c_{n} \rightarrow 0$. Then

$$
\mathrm{L}(\theta)=\mathrm{A}\{t(\theta) ; m(\theta), M(\theta)\}
$$

for almost every $\theta$ of $E$.

The deduction of this theorem from Theorem $A$ is obvious. Since $t_{n}(\theta)-t_{n-1}(\theta) \rightarrow 0$, every annulus $\mathrm{A}\left\{t(\theta) ; r_{1}, r_{2}\right\}$, with $m(\theta)<r_{1}<r_{2}<M(\theta)$, contains infinitely many points $t_{n}(\theta)$, and so also points of the set $\mathrm{L}(\theta)$. For every value of $\theta$ for which the set $\mathrm{L}(\theta)$ is of circular structure that set must contain all the circumferences $C\{t(\theta) ; r\}$ with $m(\theta) \leqq r \leqq M(\theta)$. This completes the proof.

Assume that for almost every $\theta \in E, m(\theta)=0$; that is, that for almost every value $\theta$ of $E$ there is a sequence $\left\{n_{k}\right\}$ (which may depend on $\theta$ ) such that

$$
t_{n_{k}}(\theta) \rightarrow t(\theta) \text {. }
$$

Let us suppose furthermore that $c_{n}$ tends to 0 . Then obviously

$$
\mathrm{L}(\theta)=\mathrm{K}\{t(\theta) ; M(\theta)\}
$$

almost everywhere in $\mathrm{E}$. This is certainly the case if the real part of the series (1.2.2) is a Fourier-Lebesgue series. For then, as is well known,

$$
\int_{0}^{2 \pi}\left|t(\theta)-t_{n}(\theta)\right|^{\mu} d \theta \rightarrow 0 \quad \text { for every } 0<\mu<1\left(^{6}\right)
$$

and so there is a sequence $\left\{n_{k}\right\}$, independent of $\theta$, such that (1.2.6) is satisfied almost everywhere.

(6) See, e.g., Zygmund [1, p. 153]. 
1.3. The results stated in the preceding section for the partial sums of power series can be extended to the arithmetic means of power series $\left({ }^{7}\right)$.

Let $\tau_{n}^{\alpha}(\theta)$ denote the $\alpha$ th Cesàro means of the series (1.2.2). By

$$
\mathrm{L}_{F}^{\alpha}(\theta)=\mathrm{L}^{\alpha}(\theta)
$$

we shall denote the set of the limit points of the sequence $\left\{\tau_{n}^{\alpha}(\theta)\right\}$.

Theorem 3. Suppose that the series (1.2.2) is summable $(C, \alpha+1)$ (where $\alpha>-1)$ at every point $\theta$ of a set $E$, to sum $t(\theta)$. Then at almost every point $\theta$ of $E$ the set $\mathrm{L}^{\alpha}(\theta)$ is of circular structure, with center $t(\theta)$.

In particular, the set $L^{\alpha}(\theta)$ is of circular structure almost everywhere in $E$, if the arithmetic means $\tau_{n}^{\alpha}(\theta)$ of the series (1.2.2) are bounded at every point $\theta$ of $E$.

That the second part of this theorem is a consequence of the first part follows at once from Theorem 2 .

1.4. Theorem 4. Suppose that the conditions of Theorem 3 are satisfied for some $\alpha \geqq 0$ and that $c_{n} \rightarrow 0$. Let

$$
m^{\alpha}(\theta)=\liminf _{n \rightarrow \infty}\left|\tau_{n}^{\alpha}(\theta)-t(\theta)\right|, \quad M^{\alpha}(\theta)=\underset{n \rightarrow \infty}{\limsup }\left|\tau_{n}^{\alpha}(\theta)-t(\theta)\right| .
$$

Then

$$
\mathrm{L}^{\alpha}(\theta)=\mathrm{A}\{t(\theta) ; m(\theta), M(\theta)\}
$$

almost everywhere in $E$.

This theorem follows in the same way from the preceding theorem as Theorem B follows from Theorem A, provided we prove that

$$
\tau_{n}^{\alpha}(\theta)-\tau_{n-1}^{\alpha}(\theta) \rightarrow 0
$$

which is immediate (cf. §5.3).

In the remainder of Part II we give a proof of a theorem (Theorem 5 below) which was already stated without proof in an earlier paper, and indicate extensions of the previous results to the case of Dirichlet series.

1.5. In this section we state without proofs a few known results from the theory of Cesàro arithmetic means. We state only the facts we shall actually need in the sequel.

Given a series

$$
\sum_{n=0}^{\infty} u_{n}
$$

( 7$)$ For the theory of Cesàro means, see Andersen [1] and Kogbetliantz [1]. All the results required for the present paper will be found in Zygmund [1]. 
and a number $\alpha$, we define numbers $s_{n}^{\alpha}(n=0,1, \cdots)$ by the equation

$$
\sum_{n=0}^{\infty} s_{n}^{\alpha} x^{n}=\frac{1}{(1-x)^{\alpha+1}} \sum_{n=0}^{\infty} u_{n} x^{n} .
$$

Let us consider the numbers $A_{n}^{\alpha}$ defined by the formula

$$
\sum_{n=0}^{\infty} A_{n}^{\alpha} x^{n}=\frac{1}{(1-x)^{\alpha+1}} .
$$

If the ratio

$$
\sigma_{n}^{\alpha}=s_{n}^{\alpha} / A_{n}^{\alpha}
$$

tends to $s$ as $n$ tends to infinity, the series (1.5.1) is said to be summable by the Cesàro method of arithmetic means of order $\alpha$, or simply summable $(C, \alpha)$, to sum $s$. The numbers $s_{n}^{\alpha}$ and $\sigma_{n}^{\alpha}$ are called respectively the Cesàro sums and the Cesàro means of order $\alpha$ of the series (1.5.1). If the ratio (1.5.2) is bounded [bounded below] as $n \rightarrow \infty$, the series (1.5.1) is said to be bounded $(C, \alpha)$ [bounded below $(C, \alpha)$ ].

From the above definitions one can easily deduce the following properties of Cesàro means.

(i) If the series (1.5.1) is summable $(C, \alpha)$, where $\alpha>-1$, to sum $s$, then the series is also summable $(C, \beta)$ to sum $s$, provided that $\beta>\alpha$.

If the series (1.5.1) is bounded [bounded below] $(C, \alpha)$, then it is also bounded [bounded below] $(C, \beta)$ for $\beta>\alpha>-1$.

(ii) If the series (1.5.1) is summable $(C, \alpha), \alpha>-1$, to sum $s$, then it is also summable by Abel's method (summable $A$ ) to $s$. In other words

$$
\lim _{z \rightarrow 1-0} \sum_{n=0}^{\infty} u_{n} z^{n}=s
$$

Here the variable $z$ tends to 1 along the real axis, but the result holds if $z$ tends to 1 from inside the circle $|z|<1$ along any non-tangential path, that is, along any path contained between two chords of the circumference $|z|=1$ passing through the point $z=1$.

(iii) If the series (1.5.1) is summable $(C, \alpha), \alpha>-1$, then $u_{n}=o\left(n^{\alpha}\right)$.

(iv) We have

$$
A_{n}^{\alpha}=\frac{(\alpha+1)(\alpha+2) \cdots(\alpha+n)}{n !} \simeq \frac{n^{\alpha}}{\Gamma(\alpha+1)},
$$

where the relation $a_{n} \simeq b_{n}$ means that $a_{n} / b_{n} \rightarrow 1$.

In particular, the numbers $A_{n}^{\alpha}$ are non-negative for $\alpha>-1$, form an increasing sequence tending to $+\infty$ when $\alpha>0$, and decrease to 0 when $-1<\alpha<0$. Moreover $A_{n}^{0}=1$ for all $n$. 
(v) The numbers $A_{n}^{\alpha}$ and $s_{n}^{\alpha}$ satisfy the following relations

$$
\sum_{\nu=0}^{n} A_{\nu}^{\alpha} A_{n-\nu}^{\beta}=A_{n}^{\alpha+\beta+1}, \quad \sum_{\nu=0}^{n} s_{\nu}^{\alpha} A_{n-\nu}^{\beta}=s_{n}^{\alpha+\beta+1} .
$$

In particular,

and so

$$
\sum_{\nu=0}^{n} A_{\nu}^{\alpha}=A_{n}^{\alpha+1}, \quad \sum_{\nu=0}^{n} s_{\nu}^{\alpha}=s_{n}^{\alpha+1}
$$

$$
A_{n}^{\alpha+1}-A_{n-1}^{\alpha+1}=A_{n}^{\alpha}, \quad s_{n}^{\alpha+1}-s_{n-1}^{\alpha+1}=s_{n}^{\alpha} .
$$

(vi) If $\left\{\epsilon_{\nu}\right\}$ is any sequence tending to 0 , then

$$
\sum_{\nu=0}^{n} \epsilon_{\nu} A_{\nu}^{\alpha} A_{n-\nu}^{\beta}=o\left(A_{n}^{\alpha+\beta+1}\right) \quad \text { for } \alpha>-1, \beta>-1 .
$$

This follows from (i) and from the second formula (1.5.3), if we put $\epsilon_{\nu} A_{\nu}^{\alpha}=s_{\nu}^{\alpha}$, and observe that the series summable $(C, \alpha)$ to 0 is also summable $(C, \alpha+\beta+1)$ to 0 .

(vii) It is easy to see that (1.5.4) holds if we replace the upper limit of summation $n$ by $n-k$, where $k$ is any fixed positive integer. Hence

$$
\sum_{\nu=0}^{n-k} o\left(A_{\nu}^{\alpha}\right) A_{n-\nu}^{\beta}=o\left(\dot{A}_{n}^{\alpha+\beta+1}\right) \quad \text { for } \alpha, \beta>-1, k=\text { const. }
$$

\section{PART I}

\section{LemMAS ON SUMmaBiLITy $\left(C^{*}, \alpha\right)$}

2.1. Let $\sigma_{n}^{\alpha}(\theta)$ denote the $\alpha$ th Cesàro means of the series

$$
\frac{1}{2} a_{0}+\sum_{\nu=1}^{\infty}\left(a_{\nu} \cos \nu \theta+b_{\nu} \sin \nu \theta\right) \text {. }
$$

We shall say that this series is summable $\left(C^{*}, \alpha\right)$ at the point $\theta_{0}$ to sum $s$, if

$$
\underset{\sigma_{n}}{\alpha}\left(\theta_{0}+h_{n}\right) \rightarrow s
$$

for every sequence $h_{n}=O(1 / n)$. This property is equivalent to the following one: to every constant $A>0$ and to every $\epsilon>0$ corresponds an integer $n_{0}=n_{0}(A, \epsilon)$ such that

$$
\left|\sigma_{n}^{\alpha}\left(\theta_{0}+h\right)-s\right| \leqq \epsilon \quad \text { for }|h| \leqq A / n, n>n_{0} .
$$

Lemma A. A necessary and sufficient condition that the series

$$
\sum_{\nu=0}^{\infty} a_{\nu}
$$


should be summable $(C, \alpha)$, where $\alpha>-1$, to sum $s$ is that the series

$$
\sum_{\nu=0}^{\infty} a_{\nu} \cos \nu \theta
$$

should be summable $\left(C^{*}, \alpha\right)$ at the point 0 to sum $s\left(^{8}\right)$.

Proof. The sufficiency of the condition is evident. For the proof of the necessity we may assume that $s=0$. We begin with the case of integral $\alpha$, so that $\alpha=0,1,2, \cdots$.

We write

$$
\mu_{\nu}=e^{i \nu \theta}, \quad \epsilon_{\nu}=\epsilon_{\nu}^{(n)}=A_{n-\nu}^{\alpha} \mu_{\nu} .
$$

Let $t_{n}^{\alpha}$ denote the $\alpha$ th Cesàro sums of the series (2.1.3), and let $\sigma_{n}^{\alpha}(\theta)$ be the $\alpha$ th Cesàro means of the series

$$
\sum_{\nu=0}^{\infty} a_{\nu} e^{i \nu \theta}
$$

It is sufficient to prove that, if $t_{n}^{\alpha}=o\left(n^{\alpha}\right)$, then

$$
\sigma_{n}^{\alpha}(\theta)=o(1) \quad \text { for }|n \theta| \leqq A .
$$

Applying Abel's transformation $\alpha+1$ times, we may write

$$
A_{n}^{\alpha \sigma_{n}^{\alpha}}(\theta)=\sum_{\nu=0}^{n} a_{\nu} \epsilon_{\nu}=\sum_{\nu=0}^{n-\alpha-1} t_{\nu}^{\alpha} \Delta^{\alpha+1} \epsilon_{\nu}+\sum_{k=0}^{\alpha} t_{n-k}^{k} \Delta^{k} \epsilon_{n-k}=P_{n}+Q_{n}\left({ }^{9}\right),
$$

say. The numbers $\mu_{\nu}$ being bounded, the expressions $\Delta^{k} \epsilon_{n-k}$ are also bounded for $k=0,1, \cdots, \alpha$. Since the relation $t_{n}^{\alpha}=o\left(n^{\alpha}\right)$ implies

$$
\stackrel{k}{t_{n-k}}=o\left(n^{\alpha}\right) \text { for } n \rightarrow \infty, \text { and } k=0,1, \cdots, \alpha,
$$

it follows that

$$
Q_{n}=o\left(n^{\alpha}\right) \text {. }
$$

In order to estimate the expression $P_{n}$, we use the formula

$$
\Delta^{l} \xi_{n} \eta_{n}=\sum_{j=0}^{l} C_{l, j} \Delta^{j} \xi_{n} \Delta^{l-j} \eta_{n+j}, \quad l=0,1, \cdots .
$$

Hence, taking into account that

$\left.{ }^{8}\right)$ Lemma A of the present paper is a special case of Lemma D. The latter lemma is stated in Plessner [1], for $\alpha=0,1,2, \cdots$.

( $\left.{ }^{9}\right)$ We use the notation

$$
\Delta c_{\nu}=c_{\nu}-c_{\nu+1}, \quad \Delta^{k} c_{\nu}=\Delta\left(\Delta^{k-1} c_{\nu}\right) .
$$




$$
\Delta \mu_{\nu}=e^{i \nu \theta}-e^{i(\nu+1) \theta}=e^{i(\nu+1 / 2) \theta} \cdot\left(-2 i \sin \frac{1}{2} \theta\right),
$$

and generally

$$
\Delta^{j} \mu_{\nu}=e^{i(\nu+j / 2) \theta}\left(-2 i \sin \frac{1}{2} \theta\right)^{i},
$$

we obtain

$$
\begin{aligned}
\Delta^{\alpha+1} \epsilon_{\nu} & =\sum_{j=0}^{\alpha+1} C_{\alpha+1, j} \Delta_{\nu}^{j}\left(A_{n-\nu}^{\alpha}\right) \Delta^{\alpha+1-j} \mu_{\nu+j} \\
& =\sum_{j=0}^{\alpha} C_{\alpha+1, j} A_{n-\nu}^{\alpha-j}\left(-2 i \sin \frac{1}{2} \theta\right)^{\alpha+1-j} e^{i(\alpha+1-j) \theta / 2} e^{i \nu \theta} .
\end{aligned}
$$

It follows that $P_{n}$ is equal to the sum of the expressions

$$
e^{i(\alpha+1-j) \theta / 2}\left(-2 i \sin \frac{1}{2} \theta\right)^{\alpha+1-j} C_{\alpha+1, j} \sum_{\nu=0}^{n-\alpha-1} t_{\nu}^{\alpha} A_{n-\nu}^{\alpha-j} e^{i \nu \theta}
$$

for $j=0,1, \cdots, \alpha$. The absolute value of (2.1.9) does not exceed (cf. (1.5.5))

$$
|\theta|^{\alpha+1-j} C_{\alpha+1, j} \sum_{\nu=0}^{n-\alpha-1} o\left(A_{\nu}^{\alpha}\right) A_{n-\nu}^{\alpha-j}=|\theta|^{\alpha+1-j} o\left(n^{2 \alpha-j+1}\right)=o\left(n^{\alpha}\right),
$$

provided that $|n \theta| \leqq A$. Hence $P_{n}=o\left(n^{\alpha}\right)$, and since the same relation was obtained for $Q_{n}, P_{n}+Q_{n}=o\left(n^{\alpha}\right)$. This and (2.1.7) give (2.1.6), so that the lemma is established in the case of integral $\alpha$.

2.2. In the case of fractional $\alpha>-1$, we write

$$
\beta=[\alpha]+1, \quad \mu_{\nu}=e^{i \nu \theta}, \quad \eta_{\nu}=\eta_{\nu}^{(n)}(\theta)=A_{n-\nu}^{\alpha}\left(\mu_{\nu}-\mu_{n}\right) .
$$

Hence $\beta$ is a non-negative integer greater than $\alpha$. We write

$$
A_{n}^{\alpha} \sigma_{n}^{\alpha}(\theta)=\mu_{n} \sum_{\nu=0}^{n} A_{n-\nu}^{\alpha} a_{\nu}+\sum_{\nu=0}^{n} a_{\nu} \eta_{\nu}=o\left(n^{\alpha}\right)+\sum_{\nu=0}^{n} a_{\nu} \eta_{\nu}
$$

To the last sum we apply Abel's transformation $\beta+1$ times:

$$
\sum_{\nu=0}^{n} a_{\nu} \eta_{\nu}=\sum_{\nu=0}^{n-\beta-1} t_{\nu}^{\beta} \Delta^{\beta+1} \eta_{\nu}+\sum_{k=1}^{\beta} t_{n-k}^{k} \Delta^{k} \eta_{n-k}=P_{n}+Q_{n}
$$

say. Now

$$
\begin{aligned}
\Delta^{k} \eta_{\nu} & =\sum_{j=0}^{k-1} C_{k, j} A_{n-\nu}^{\alpha-j} \Delta^{k-j} \mu_{\nu+j}+A_{n-\nu}^{\alpha-k}\left(\mu_{\nu+k}-\mu_{n}\right) \\
& =\sum_{j=0}^{k-1} C_{k, j} A_{n-\nu}^{\alpha-j} \Delta^{k-j}{ }_{\mu_{\nu+j}}+O(|\theta|)
\end{aligned}
$$


for $\nu=n-k$ and $k=1,2, \cdots, \beta$. Since the series (2.1.3) is summable $(C, \alpha)$ and so also $(C, \beta)$ to 0 , we obtain

Hence

$$
t_{n-k}^{k}=o\left(n^{\beta}\right) \quad \text { for } k=0,1, \cdots .
$$

$$
Q_{n}=\sum_{k=1}^{\beta} o\left(n^{\beta}\right) O(|\theta|)=o\left(n^{\beta-1}\right)=o\left(n^{\alpha}\right)
$$

for $|n \theta| \leqq A$.

It remains to estimate the sum $P_{n}$. On account of the first equation (2.2.3) with $k=\beta+1, P_{n}$ is a sum of $\beta+1$ expressions

$$
C_{\beta+1, j} \sum_{\nu=0}^{n-\beta-1} t_{\nu}^{\beta} A_{n-\nu}^{\alpha-j} \Delta^{\beta+1-j} \mu_{\nu+j}, \quad j=0,1, \cdots, \beta,
$$

and of the expression

$$
\sum_{\nu=0}^{n-\beta-1} t_{\nu}^{\beta} A_{n-\nu}^{\alpha-\beta-1}\left(\mu_{\nu+\beta+1}-\mu_{n}\right)
$$

The sum (2.2.5) is

$$
O\left(|\theta|^{\beta+1-j}\right) \sum_{\nu=0}^{n-\beta-1} o\left(A_{\nu}^{\beta}\right) A_{n-\nu}^{\alpha-j}=O\left(n^{j-\beta-1}\right) o\left(n^{\alpha+\beta-j+1}\right)=o\left(n^{\alpha}\right),
$$

and the sum (2.2.6) does not exceed in absolute value

$$
\begin{aligned}
\sum_{\nu=0}^{n-\beta-1} o\left(A_{\nu}^{\beta}\right) A_{n-\nu}^{\alpha-\beta-1}(n-\nu)|\theta| & =O(|\theta|) \sum_{\nu=0}^{n-\beta-1} o\left(A_{\nu}^{\beta}\right) A_{n-\nu}^{\alpha-\beta} \\
& =O(|\theta|) o\left(n^{\alpha+1}\right)=o\left(n^{\alpha}\right) .
\end{aligned}
$$

It follows that

$$
P_{n}=o\left(n^{\alpha}\right) \text {. }
$$

From (2.2.1), (2.2.2), (2.2.4), (2.2.7) we deduce (2.1.6), and so the proof of Lemma $\mathrm{A}$ is complete.

2.3. Lemma B. A necessary and sufficient condition that the series

$$
\sum_{v=0}^{\infty} b_{v} \sin \nu \theta
$$

should be summable $\left(C^{*}, \alpha\right), \alpha>-1$, at the point $\theta=0$, is that the sequence $\left\{\nu b_{\nu}\right\}(\nu=0,1,2, \cdots)$ should be summable $(C, \alpha+1)$ to 0 .

Proof. The argument is similar to that of Lemma A. We begin by proving the sufficiency of the condition, and we assume first that $\alpha$ is a non-negative integer. Let 


$$
\nu b_{\nu}=c_{\nu}, \quad \mu_{\nu}=\mu_{\nu}^{(n)}(\theta)=\frac{\sin \nu \theta}{\nu \theta}, \quad \gamma_{\nu}=\gamma_{\nu}^{(n)}(\theta)=A_{n-\nu}^{\alpha} \mu_{\nu} .
$$

By $\sigma_{n}^{\alpha}(\theta)$ we shall denote the $\alpha$ th Cesàro means of the series (2.3.1) and by $u_{n}^{k}$ the iterated sums of the sequence $\left\{c_{\nu}\right\}$, so that

$$
\begin{array}{r}
u_{n}=u_{n}^{0}=c_{n}, \quad u_{n}^{k}=u_{0}^{k-1}+u_{1}^{k-1}+\cdots+u_{n}^{k-1}, \\
n=0,1, \cdots ; k=1,2, \cdots .
\end{array}
$$

Hence

$$
A_{n}^{\alpha \alpha} \sigma_{n}^{\alpha}(\theta)=\theta \sum_{\nu=0}^{n} A_{n-\nu}^{\alpha} \nu b_{\nu} \frac{\sin \nu \theta}{\nu \theta}=\theta \sum_{\nu=0}^{n} c_{\nu} \gamma_{\nu} .
$$

Applying Abel's transformation $\alpha+1$ times we have

$$
\theta \sum_{\nu=0}^{n} c_{\nu} \gamma_{\nu}=\theta \sum_{\nu=0}^{n-\alpha-1} u_{\nu}^{\alpha+1} \Delta^{\alpha+1} \gamma_{\nu}+\theta \sum_{k=0}^{\alpha} u_{n-k}^{k+1} \Delta^{k} \gamma_{n-k}=\theta P_{n}+\theta Q_{n},
$$

say. By hypothesis, $u_{n}^{\alpha+1}=o\left(n^{\alpha+1}\right)$, and so also $u_{n}^{k+1}=o\left(n^{\alpha+1}\right)$ for $k=0,1, \cdots, \alpha$. This gives

$$
\theta Q_{n}=\theta o\left(n^{\alpha+1}\right)=o\left(n^{\alpha}\right)
$$

if only $|n \theta| \leqq A$. Since

$$
\Delta^{\alpha+1} \gamma_{\nu}=\sum_{j=0}^{\alpha+1} C_{\alpha+1, j} \Delta_{\nu}^{j} A_{n-\nu}^{\alpha} \Delta^{\alpha+1-j} \mu_{\nu+j}=\sum_{j=0}^{\alpha} C_{\alpha+1, j} A_{n-\nu}^{\alpha-j} O\left(|\theta|^{\alpha+1-j}\right)\left({ }^{10}\right),
$$

$\theta P_{n}$ is a sum of $\alpha+1$ terms of the form

$$
O\left(|\theta|^{\alpha+2-j}\right) \sum_{\nu=0}^{n-\alpha-1} o\left(A_{\nu}^{\alpha+1}\right) A_{n-\nu}^{\alpha-j}=O\left(|\theta|^{\alpha+2-j}\right) o\left(n^{2 \alpha-j+2}\right),
$$

where $j=0,1, \cdots, \alpha$. Hence, if $|n \theta| \leqq A$,

$$
\theta P_{n}=o\left(n^{\alpha}\right) \text {. }
$$

From (2.3.3), (2.3.4), (2.3.5) and (2.3.6) we see that

$$
\sigma_{n}^{\alpha}(\theta) \rightarrow 0 \quad \text { for } n \rightarrow \infty \text { and }|n \theta| \leqq A .
$$

This completes the proof of sufficiency in Lemma B for the case of integral $\alpha$.

2.4. If $\alpha$ is fractional, we write

(10) Here (and in the sequel) we use the known fact that if a function $\phi(u)$ has a $p$ th derivative, and if $\phi(\nu)=\phi_{\nu}$, then

$$
\Delta^{p} \phi_{\nu}=(-1)^{p} \phi(\nu+\theta p)
$$

where $\theta$ is a number contained between 0 and 1 . 


$$
\beta=[\alpha]+1, \quad \xi_{\nu}=\xi_{\nu}^{(n)}(\theta)=A_{n-\nu}^{\alpha}\left(\mu_{\nu}-\mu_{n}\right),
$$

where $\mu_{\nu}$ is defined by (2.3.2). Then

First

$$
A_{n}^{\alpha} \sigma_{n}^{\alpha}(\theta)=\theta \mu_{n} \sum_{\nu=0}^{n} A_{n-\nu}^{\alpha} c_{\nu}+\theta \sum_{\nu=0}^{n} c_{\nu} \xi_{\nu}
$$

$$
\theta \mu_{n} \sum_{\nu=0}^{n} A_{n-\nu}^{\alpha} c_{\nu}=\theta \mu_{n} O\left(n^{\alpha+1}\right)=o\left(n^{\alpha}\right)
$$

for $|n \theta| \leqq A$. Now

$$
\theta \sum_{\nu=0}^{n} c_{\nu} \xi_{\nu}=\theta \sum_{\nu=0}^{n-\beta-1} u_{\nu}^{\beta+1} \Delta^{\beta+1} \xi_{\nu}+\theta \sum_{k=1}^{\beta} u_{n-k}^{k+1} \Delta^{k} \mu_{n-k}=\theta P_{n}+\theta Q_{n},
$$

say. But

$$
\begin{aligned}
\Delta^{k} \xi_{\nu} & =\sum_{j=0}^{k-1} C_{k, j} A_{n-\nu}^{\alpha-j} \Delta^{k-j} \mu_{\nu+j}+A_{n-\nu}^{\alpha-k}\left(\mu_{\nu+k}-\mu_{n}\right) \\
& =\sum_{j=0}^{k-1} C_{k, j} A_{n-\nu}^{\alpha-j} \Delta^{k-j} \mu_{\nu+j}=O(|\theta|)
\end{aligned}
$$

for $k=1,2, \cdots, \beta$ and $\nu=n-k$. By hypothesis, the sequence $\left\{c_{\nu}\right\}$ is summable $(C, \alpha+1)$, and so also $(C, \beta+1)$, to 0 . Hence $u_{n-k}^{k+1}=o\left(n^{\beta+1}\right)$ for $k=1,2, \cdots, \beta$. This gives

$$
\theta Q_{n}=\theta \cdot O(|\theta|) o\left(n^{\beta+1}\right)=o\left(n^{\beta-1}\right)=o\left(n^{\alpha}\right)
$$

for $|n \theta| \leqq A$.

Substituting into the formula for $P_{n}$ the first formula (2.4.3) with $k=\beta+1$, we see that $\theta P_{n}$ is a sum of $\beta+1$ expressions

$$
\theta C_{\beta+1, j} \sum_{\nu=0}^{n-\beta-1} o\left(A_{\nu}^{\beta+1}\right) A_{n-\nu}^{\alpha-j} \Delta^{\beta+1-j} \mu_{\nu+j}, \quad j=0,1, \cdots, \beta,
$$

and of the expression

$$
\theta \sum_{\nu=0}^{n-\beta-1} o\left(A_{\nu}^{\beta+1}\right) A_{n-\nu}^{\alpha-\beta-1}\left(\mu_{\nu+\beta+1}-\mu_{n}\right) .
$$

The absolute value of $(2.4 .5)$ is

$$
O\left(|\theta|^{\beta+2-j}\right) \sum_{\nu=0}^{n-\beta-1} o\left(A_{\nu}^{\beta+1}\right) A_{n-\nu}^{\alpha-j}=O\left(|\theta|^{\beta+2-j}\right) o\left(n^{\alpha+\beta-j+2}\right)=o\left(n^{\alpha}\right),
$$

and the absolute value of (2.4.6) is

$$
O\left(\theta^{2}\right) \sum_{\nu=0}^{n-\beta-1} o\left(A_{\nu}^{\beta+1}\right) O\left(A_{n-\nu}^{\alpha-\beta}\right)=O\left(\theta^{2}\right) o\left(n^{\alpha+2}\right)=o\left(n^{\alpha}\right) .
$$


Hence $\theta P_{n}=o\left(n^{\alpha}\right)$, which in view of (2.4.1), (2.4.2) and (2.4.4) gives (2.3.7).

2.5. In order to prove the necessity of the condition in Lemma $B$, we have to show that, if the series (2.3.1) is summable $\left(C^{*}, \alpha\right)$ at the point $\theta=0$, then the expression

$$
\sum_{\nu=0}^{n} A_{n-\nu}^{\alpha} \nu b_{\nu}=\frac{1}{\theta} \sum_{\nu=0}^{n} A_{n-\nu}^{\alpha} b_{\nu} \sin \nu \theta \cdot \frac{\nu \theta}{\sin \nu \theta}
$$

is $o\left(n^{\alpha+1}\right)$. We shall prove a slightly more general result, which will be required later on and which may be stated in the form of the following

Lemma C. Let $\left\{h_{n}\right\}$ be a sequence of numbers tending to 0 and satisfying for $n>n_{0}$ the condition

$$
0<\delta \leqq n h_{n} \leqq \pi-\delta,
$$

where $\delta$ is any fixed positive number less than $\frac{1}{2} \pi$. If for some such sequence the Cesaro means $\sigma_{n}^{\alpha}(\theta)$, where $\alpha>-1$, of the series (2.3.1) satisfy the condition

$$
\sigma_{\nu}^{\alpha}\left(h_{n}\right) \rightarrow 0
$$$$
\text { for } \nu \rightarrow \infty \text { and } n \geqq \nu
$$

then the sequence $\left\{\nu b_{\nu}\right\}$ is summable $(C, \alpha+1)$ to 0 .

Proof. The argument does not differ appreciably from that of the preceding lemmas. We write

$$
\begin{aligned}
\mu_{\nu} & =\mu_{\nu}(\theta)=\frac{\nu \theta}{\sin \nu \theta}, & \epsilon_{\nu} & =\epsilon_{\nu}^{(n)}=A_{n-\nu}^{\alpha} \mu_{\nu}\left(h_{n}\right), \\
v_{n}(\theta) & =v_{n}^{0}(\theta)=\sum_{\nu=0}^{n} b_{\nu} \sin \nu \theta, & v_{n}^{k}(\theta)=\sum_{\nu=0}^{n} v_{\nu}^{k-1}(\theta), & k=1,2, \cdots,
\end{aligned}
$$

and we suppose first that $\alpha$ is an integer. If we substitute $\theta=h_{n}$ into the righthand side of (2.5.1) and denote the resulting expression by $J_{n}$, then (for $n>n_{0}$ )

$$
\begin{aligned}
J_{n} & =h_{n}^{-1} \sum_{\nu=0}^{n} \epsilon_{\nu} b_{\nu} \sin \nu h_{n} \\
& =h_{n}^{-1} \sum_{\nu=0}^{n-\alpha-1} v_{\nu}^{\alpha}\left(h_{n}\right) \Delta^{\alpha+1} \epsilon_{\nu}+h_{n}^{-1} \sum_{k=0}^{\alpha} v_{n-k}^{k}\left(h_{n}\right) \Delta^{k} \epsilon_{n-k}=h_{n}^{-1} P_{n}+h_{n}^{-1} Q_{n},
\end{aligned}
$$

say. From (2.5.3) we see that $v_{\nu}^{\alpha}\left(h_{n}\right)=o\left(\nu^{\alpha}\right)=o\left(n^{\alpha}\right)$. Hence

$$
v_{\nu}^{k}\left(h_{n}\right)=o\left(n^{\alpha}\right) \quad \text { for } \nu \rightarrow+\infty, n \geqq \nu, k=0,1, \cdots, \alpha_{r}
$$

and so (cf. (2.5.2))

$$
h_{n}^{-1} Q_{n}=O(n) \sum_{k=0}^{\alpha} o\left(n^{\alpha}\right) \cdot O(1)=o\left(n^{\alpha+1}\right) .
$$


Since

$$
\Delta^{\alpha+1} \epsilon_{\nu}=\sum_{j=0}^{\alpha+1} C_{\alpha+1, j} A_{n-\nu}^{\alpha-j} \Delta_{\nu}^{\alpha+1-j} \mu_{\nu+j}\left(h_{n}\right),
$$

$h_{n}^{-1} P_{n}$ is a sum of expressions

$$
O\left(\left|h_{n}\right|^{\alpha-j}\right) \sum_{\nu=0}^{n-\alpha-1} o\left(A_{\nu}^{\alpha}\right) A_{n-\nu}^{\alpha-j}=o\left(n^{\alpha+1}\right), \quad j=0,1, \cdots, \alpha+1 .
$$

From this and from (2.5.5) and (2.5.6) we see that the left-hand side of (2.5.1) is $o\left(n^{\alpha+1}\right)$, which proves Lemma $\mathrm{C}$ in the case of integral $\alpha$.

2.6. If $\alpha$ is fractional, we write

$$
\beta=[\alpha]+1, \quad \eta_{\nu}=\eta_{\nu}^{(n)}=A_{n-\nu}^{\alpha}\left(\mu_{\nu}\left(h_{n}\right)-\mu_{n}\left(h_{n}\right)\right),
$$

where $\mu_{\nu}$ has the same meaning as in (2.5.4). We get

$$
\begin{aligned}
J_{n} & =h_{n}^{-1} \mu_{n}\left(h_{n}\right) \sum_{\nu=0}^{n} A_{n-\nu}^{\alpha} b_{\nu} \sin \nu h_{n}+h_{n}^{-1} \sum_{\nu=0}^{n} \eta_{\nu} b_{\nu} \sin \nu h_{n} \\
& =o\left(n^{\alpha+1}\right)+h_{n}^{-1} \sum_{\nu=0}^{n} \eta_{\nu} b_{\nu} \sin \nu h_{n} .
\end{aligned}
$$

Furthermore,

$$
\begin{aligned}
h_{n}^{-1} \sum_{\nu=0}^{n} \eta_{\nu} b_{\nu} \sin \nu h_{n} & =h_{n}^{-1} \sum_{\nu=0}^{n-\beta-1} v_{\nu}^{\beta}\left(h_{n}\right) \Delta^{\beta+1} \eta_{\nu}+h_{n}^{-1} \sum_{k=1}^{\beta} v_{n-k}^{k}\left(h_{n}\right) \Delta^{k} \eta_{n-k} \\
& =h_{n}^{-1} P_{n}+h_{n}^{-1} Q_{n} .
\end{aligned}
$$

From (2.5.3) and from the inequality $\beta>\alpha$ we get

$$
v_{\nu}^{\beta}\left(h_{n}\right)=o\left(n^{\beta}\right) \quad \text { for } \nu \rightarrow \infty, n \geqq \nu,
$$

whence

$$
v_{\nu}^{k}\left(h_{n}\right)=o\left(n^{\beta}\right) \quad \text { for } \nu \rightarrow \infty, n \geqq \nu, k=0,1, \cdots, \beta .
$$

Now

$$
\Delta^{k} \eta_{\nu}=\sum_{j=0}^{k-1} C_{k, j} A_{n-\nu}^{\alpha-j} \Delta_{\nu}^{k-j} \mu_{\nu+j}\left(h_{n}\right)+A_{n-\nu}^{\alpha-k}\left(\mu_{\nu+k}\left(h_{n}\right)-\mu_{n}\left(h_{n}\right)\right)
$$

so that

$$
h_{n}^{-1} Q_{n}=h_{n}^{-1} \sum_{k=1}^{\beta} o\left(n^{\beta}\right) O\left(n^{-1}\right)=o\left(n^{\beta}\right)=o\left(n^{\alpha+1}\right) .
$$


If we substitute $k=\beta+1$ into (2.6.1), and observe that $h_{n}^{-1} P_{n}$ is correspondingly a sum of expressions

$$
h_{n}^{-1} \sum_{\nu=0}^{n-\beta-1} o\left(A_{n}^{\beta}\right) A_{n-\nu}^{\alpha-i} O\left(n^{j-\beta-1}\right)=o\left(n^{\alpha+1}\right), \quad j=0,1, \cdots, \beta,
$$

and of the expression

$$
h_{n}^{-1} \sum_{\nu=0}^{n-\beta-1} o\left(A_{n}^{\beta}\right) A_{n-\nu}^{\alpha-\beta-1} O\left((n-\nu) h_{n}\right)=o\left(n^{\alpha+1}\right)
$$

we easily get

$$
J_{n}=o\left(n^{\alpha+1}\right),
$$

which completes the proof of Lemma B as well as that of Lemma C.

2.7. Lemma $\mathrm{D}\left({ }^{11}\right)$. Let $\sigma_{n}^{\alpha}(\theta)$ denote the Cesàro means of the series

$$
\frac{1}{2} a_{0}+\sum_{\nu=1}^{\infty}\left(a_{\nu} \cos \nu \theta+b_{\nu} \sin \nu \theta\right) \text {. }
$$

$A$ necessary and sufficient condition that this series should at the point $\theta_{0}$ be summable $\left(C^{*}, \alpha\right)$, where $\alpha>-1$, to sum $g$, is that

(i) this series should be summable $(C, \alpha)$ at the point $\theta_{0}$ to sum $g$;

(ii) there should exist a sequence $\left\{h_{n}\right\}$ satisfying for $n>n_{0}$ the condition

$$
0<\delta \leqq\left|n h_{n}\right| \leqq \pi-\delta
$$

$\delta$ independent of $n$,

and such that

$$
\sigma_{\nu}^{\alpha}\left(\theta_{0}+h_{n}\right) \rightarrow g, \quad \nu \rightarrow \infty, n \geqq \nu .
$$

Proof. That the condition is necessary is obvious. In order to prove its sufficiency we may suppose that $\theta_{0}=0$. By hypothesis, the series

$$
\frac{1}{2} a_{0}+\sum_{\nu=1}^{\infty} a_{\nu}
$$

is summable $(C, \alpha)$ to $g$, and so on account of Lemma A the cosine part of the series (2.7.1) is summable $\left(C^{*}, \alpha\right)$ to $g$ at the point $\theta=0$. Hence, the sine part of (2.7.1) satisfies the hypothesis of Lemma C. It follows that the sequence $\left\{n b_{n}\right\}$ is summable $(C, \alpha+1)$ to 0 . Consequently (Lemma B), the sine part of (2.7.1) is summable $\left(C^{*}, \alpha\right)$ to 0 at the point $\theta=0$, and so the series (2.7.1) is summable $\left(C^{*}, \alpha\right)$ to $g$ at that point. This completes the proof of Lemma D.

2.8. Lemma E. If the series (2.7.1) is summable $\left(C^{*}, \alpha\right), \alpha>-1$, at a point $\theta_{0}$, then the sequence

(1i) Cf. Footnote 8. 


$$
n B_{n}\left(\theta_{0}\right)=n\left(b_{n} \cos n \theta_{0}-a_{n} \sin n \theta_{0}\right)
$$

is summable $(C, \alpha+1)$ to 0 .

Assuming for simplicity that $\theta_{0}=0$, we see at once that Lemma $E$ is a consequence of Lemmas $\mathrm{A}$ and $\mathrm{B}\left({ }^{12}\right)$.

\section{Summability $A^{*}$. Lemmas on NUMERICAL SERIES}

3.1. We shall say that the series

$$
\frac{1}{2} a_{0}+\sum_{\nu=1}^{\infty}\left(a_{\nu} \cos \nu \theta+b_{\nu} \sin \nu \theta\right)
$$

is summable $A^{*}$ at a point $\theta_{0}$ to sum $g$, if the harmonic function

$$
\frac{1}{2} a_{0}+\sum_{\nu=1}^{\infty}\left(a_{\nu} \cos \nu \theta+b_{\nu} \sin \nu \theta\right) r^{\nu}
$$

tends to $g$ when the point $r e^{i \theta}$ tends to $e^{i \theta_{0}}$ along any non-tangential path.

Lemma F. If the series (3.1.1) is summable $\left(C^{*}, \alpha\right), \alpha>-1$, at a point $\theta_{0}$ to sum $g$, it is also summable $A^{*}$ at that point to $g$.

Proof. Let us assume that $\theta_{0}=0$. From the hypothesis of Lemma $F$ it follows that both the series

$$
\frac{1}{2} a_{0}+\sum_{\nu=1}^{\infty} a_{\nu} \cos \nu \theta, \quad \sum_{\nu=0}^{\infty} b_{\nu} \sin \nu \theta
$$

are summable $\left(C^{*}, \alpha\right)$ at the point $\theta$, the sum of the first of these series being $g$, the sum of the second being 0 . It is sufficient to show that

$$
\frac{1}{2} a_{0}+\sum_{\nu=1}^{\infty} a_{\nu} r^{\nu} \cos \nu \theta \rightarrow g, \quad \sum_{\nu=1}^{\infty} b_{\nu} \gamma^{\nu} \sin \nu \theta \rightarrow 0,
$$

provided that

$$
z \rightarrow 1, \quad|1-z| \leqq C(1-r)
$$
where $z=r e^{i \theta}$.

Now,

$$
\frac{1}{2} a_{0}+\sum_{\nu=1}^{\infty} a_{\nu} r^{\nu} \cos \nu \theta=\Re\left(\frac{1}{2} a_{0}+\sum_{\nu=1}^{\infty} a_{\nu} z^{\nu}\right),
$$

(12) From Lemmas $\mathrm{E}$ and $\mathrm{G}$ (see below) it follows at once that if the series (1.1.1) is summable $\left(C^{*}, \alpha\right), \alpha>-1$, at a point $\theta_{0}$, and if the series (1.1.2) is summable $A$ at that point, then the series (1.1.2) is also summable $\left(C^{*}, \alpha\right)$ at the point $\theta_{0}$.

This result, with summability $A$ replaced by summability $C$, and with $\alpha=0,1, \cdots$, is stated by Plessner [1]. 
and since the series $\frac{1}{2} a_{0}+a_{1}+a_{2}+\cdots$ is summable $(C, \alpha)$ to $g$, the very well known extension of the Abel-Stolz lemma gives $\left({ }^{13}\right)$ that, under the conditions (3.1.3), the function

$$
\frac{1}{2} a_{0}+a_{1} z+a_{2} z^{2}+\cdots
$$

tends to $g$. In yiew of (3.1.4), we get the first relation (3.1.2) under the same conditions.

Furthermore, denoting by $u_{\nu}^{\alpha}$ the $\alpha$ th Cesàro means of the sequence $\left\{\nu b_{\nu}\right\}$, where $\nu=0,1, \cdots$, we may write

$$
\begin{aligned}
\sum_{\nu=1}^{\infty} b_{\nu} r^{\nu} \sin \nu \theta & =\int_{0}^{\theta}\left(\sum_{\nu=0}^{\infty} \nu b_{\nu} r^{\nu} \cos \nu t\right) d t=\int_{0}^{\theta} \Re\left(\sum_{\nu=0}^{\infty} \nu b_{\nu} \zeta^{\nu}\right) d t \\
& =\int_{0}^{\theta} \Re\left\{\left(\sum_{\nu=0}^{\infty} u_{\nu}^{\alpha+1} \zeta^{\nu}\right)(1-\zeta)^{\alpha+1}\right\} d t,
\end{aligned}
$$

where $\zeta=r e^{i t}$. Taking into account that

$$
|\zeta|=r, \quad|1-\zeta| \leqq C(1-r), \quad u_{\nu}^{\alpha+1}=o\left(\nu^{\alpha+1}\right),
$$

we see that the absolute value of the last integral does not exceed

$$
|\theta| \cdot o\left(\frac{1}{(1-r)^{\alpha+2}}\right) \cdot C^{\alpha+1}(1-r)^{\alpha+1}=o(1) .
$$

This proves the second relation (3.1.2) under the conditions (3.1.3). The proof of Lemma $F$ is thus complete.

3.2. Lemma $G$ which follows is known $\left({ }^{14}\right)$. We give its proof here for the sake of completeness only.

Lemma G. If the series

$$
\sum_{\nu=0}^{\infty} a_{\nu}
$$

is summable $A$, and if the sequence $\left\{c_{\nu}\right\}=\left\{\nu a_{\nu}\right\}$ is summable $(C, \alpha+1)$ to 0 , where $\alpha>-1$, then the series (3.2.1) is summable $(C, \alpha)$.

Proof. Let

$$
\sigma_{n}^{\gamma}=\frac{s_{n}^{\gamma}}{A_{n}^{\gamma}}
$$

denote the $\gamma$ th Cesàro means of the series (3.2.1). We shall say that the series (3.2.1) is summable by the method $A(C, \gamma)$ to sum $s$, if

(13) See $\$ 1.5$ (ii).

(14) See, for example, Andersen [1]. 


$$
\lim _{r \rightarrow 1-0}(1-r) \sum_{n=0}^{\infty} \sigma_{n}^{\gamma} r^{n}=s .
$$

Summability $A(C, 0)$ is plainly identical with summability $A$. For the proof of Lemma $G$ we shall require the following three supplementary propositions $\left({ }^{15}\right)$ :

(i) If the series (3.2.1) is summable $A$ to $s$, it is also summable $A(C, \gamma)$ to $s$, provided that $\gamma \geqq 0$.

(ii) If the series (3.2.1) is summable $A(C, \gamma)$, where $\gamma>0$, to $s$, and if $a_{\nu}=o(1 / \nu)$, then the series converges to $s$.

(iii) If $a_{\nu}=o(1 / \nu)$ and if the series (3.2.1) is convergent, then it is summable $(C,-1+\epsilon)$ for every $\epsilon>0$.

In order to prove (i) we may assume that $\gamma>0$ and that $s=0$. We observe that

Hence, if

$$
\frac{1}{A_{n}^{\gamma}}=\gamma \int_{0}^{1}(1-u)^{\gamma-1} u^{n} d u \quad \gamma>0 ; n=0,1, \cdots
$$

$$
f(r)=\sum_{\nu=0}^{\infty} a_{\nu} r^{\nu} \quad \text { for } 0 \leqq r<1,
$$

then

$$
\begin{aligned}
(1-r) \sum_{n=0}^{\infty} \sigma_{n}^{\gamma} r^{n} & =(1-r) \sum_{n=0}^{\infty} \frac{s_{n}^{\gamma}}{A_{n}^{\gamma}} r^{n} \\
& =(1-r) \gamma \int_{0}^{1}(1-u)^{\gamma-1} \sum_{n=0}^{\infty} s_{n}^{\gamma}(u r)^{n} d u \\
& =(1-r) \gamma \int_{0}^{1} \frac{f(u r)}{(1-r u)^{\gamma+1}}(1-u)^{\gamma-1} d u
\end{aligned}
$$

Let $M$ denote the upper bound of $f(x)$ for $0 \leqq x<1$, and let us suppose that $|f(x)| \leqq \delta$ for $1-\epsilon \leqq x<1$. The last expression in (3.2.2) does not exceed in absolute value

$$
M(1-r) \gamma \int_{0}^{(1-\epsilon) r^{-1}} \frac{(1-u)^{\gamma-1}}{(1-r u)^{\gamma+1}} d u+\delta(1-r) \gamma \int_{(1-\epsilon) r-1}^{1} \frac{(1-u)^{\gamma-1}}{(1-r u)^{\gamma+1}} d u .
$$

Here the first term tends to 0 as $r \rightarrow 1$, and the second term does not exceed

$$
\delta(1-r) \gamma \int_{0}^{1} \frac{(1-u)^{\gamma-1}}{(1-r u)^{\gamma+1}} d u=\delta
$$

(15) Propositions (i) and (ii) are taken from Zygmund [1]. Proposition (iii) was proved by Hardy and Littlewood [1], even with $o$ replaced by $O$. 
(in order to verify the equation, it is sufficient to substitute $f(x) \equiv 1$, i.e., $\sigma_{n}^{\gamma}=1$ for $n=0,1, \cdots$, into the extreme terms of (3.2.2)). This completes the proof of (i).

In order to prove (ii), it is sufficient to show that the summability $A(C, \gamma)$ of the series (3.2.1) and the condition $a_{n}=o(1 / n)$ imply the summability $A$ of (3.2.1), for then the result will follow from Tauber's classical theorem. We may of course assume that $\gamma \neq 0$. Let $s_{n}$ denote the $n$th partial sum of the series (3.2.1). We shall show that then

$$
\sigma_{n}^{\gamma}-s_{n} \rightarrow 0
$$

for $n \rightarrow \infty$.

For

$$
\sigma_{n}^{\gamma}-s_{n}=\frac{1}{A_{n}^{\gamma}} \sum_{\nu=0}^{n}\left(A_{n-\nu}^{\gamma}-A_{n}^{\gamma}\right) a_{\nu}
$$

and

$$
A_{n}^{\gamma}-A_{n-\nu}^{\gamma}=\sum_{\mu=n-\nu+1}^{n} A_{\mu}^{\gamma-1}, \quad 0<\nu \leqq n .
$$

Hence

$$
\begin{array}{llrl}
0 \leqq A_{n}^{\gamma}-A_{n-\nu}^{\gamma} \leqq \nu A_{n}^{\gamma-1} & \text { for } & \gamma \geqq 1, \\
0 \leqq A_{n}^{\gamma}-A_{n-\nu}^{\gamma} \leqq \nu A_{n-\nu}^{\gamma-1} \text { for } & 0<\gamma \leqq 1 .
\end{array}
$$

Consequently, since $\nu a_{\nu}=o(1)$, we obtain

$$
\begin{array}{ll}
\left|\sigma_{n}^{\gamma}-s_{n}\right| \leqq \frac{A_{n}^{\gamma-1}}{A_{n}^{\gamma}} \sum_{\nu=1}^{n} o(1)=o(1), \quad \text { if } \quad \gamma \geqq 1, \\
\left|\sigma_{n}^{\gamma}-s_{n}\right| \leqq \frac{1}{A_{n}^{\gamma}} \sum_{\nu=1}^{n} o(1) A_{n-\nu}^{\gamma-1}=o(1), & \text { if } \quad 0<\gamma \leqq 1 .
\end{array}
$$

This completes the proof of (ii).

In order to prove (iii), we observe that

$$
\sigma_{n}^{-1+\epsilon}=\frac{1}{A_{n}^{-1+\epsilon}} \sum_{\nu=0}^{[n / 2]} A_{n-\nu}^{-1+\epsilon} a_{\nu}+\frac{1}{A_{n}^{-1+\epsilon}} \sum_{\nu=[n / 2]+1}^{n} A_{n-\nu}^{-1+\epsilon} a_{\nu}=P_{n}+Q_{n},
$$

say. Now

$$
\left|Q_{n}\right| \leqq \frac{1}{A_{n}^{-1+\epsilon}} \cdot o(1 / n) \cdot \sum_{\nu=0}^{n} A_{n-\nu}^{-1+\epsilon}=o(1) .
$$

Denoting by $s_{n}$ the partial sums of the series (3.2.1) and applying Abel's transformation we easily get 


$$
P_{n}=\frac{1}{A_{n}^{-1+\epsilon}} \sum_{\nu=0}^{[n / 2]-1} s_{\nu} A_{n-\nu}^{-2+\epsilon}+\frac{1}{A_{[n / 2]}^{-1+\epsilon}} s_{[n / 2]} A_{n-[n / 2]}^{-1+\epsilon} .
$$

Assuming, as we may, that $s_{\nu} \rightarrow 0$, we see that the last term on the right of (3.2.6) is $o(1)$. The first term on the right is

$$
\frac{1}{A_{n}^{-1+\epsilon}} O\left(n^{-2+\epsilon}\right) \sum_{\nu=0}^{n}\left|s_{\nu}\right|=O(1 / n) \sum_{\nu=0}^{n}\left|s_{\nu}\right|=o(1) \text {. }
$$

Hence

$$
\sigma_{n}^{-1+\epsilon} \rightarrow 0
$$

which completes the proof of (iii).

3.3. We now pass on the proof of Lemma G. We may assume that the series is summable $A$ to 0 and that $a_{0}=0$. Let $\sigma_{n}^{\alpha}=s_{n}^{\alpha} / A_{n}^{\alpha}$ denote the $\alpha$ th Cesàro means of the series (3.2.1), and let us suppose first that $\alpha$ is an integer. We write

$$
\begin{array}{rlr}
\nu a_{\nu}=c_{\nu}, & C_{\nu}^{0}=c_{\nu}, \quad C_{\nu}^{k}=\sum_{\mu=0}^{\nu} C_{\mu}^{k-1}, & \nu=0,1,2, \cdots, \\
\epsilon_{0}=0, & \epsilon_{\nu}=\epsilon_{\nu}^{(n)}=A_{n-\nu}^{\alpha} \frac{1}{\nu}, & \nu=1,2, \cdots .
\end{array}
$$

Then

$$
s_{n}^{\alpha}=\sum_{\nu=0}^{n} \epsilon_{\nu} C_{\nu}=\sum_{\nu=0}^{n-\alpha-1} C_{\nu}^{\alpha+1} \Delta^{\alpha+1} \epsilon_{\nu}+\sum_{k=0}^{\alpha} C_{n-k}^{k+1} \Delta^{k} \epsilon_{n-k}=P_{n}+Q_{n},
$$

say. From the formula

$$
\Delta^{k} \epsilon_{\nu}=\sum_{j=0}^{k} C_{k, j} A_{n-\nu}^{\alpha-j} \Delta^{k-j} \frac{1}{\nu+j}
$$

and from the relation

it is easy to deduce that

$$
C_{n}^{\alpha+1}=o\left(n^{\alpha+1}\right)
$$

$$
Q_{n}=o\left(n^{\alpha}\right) .
$$

Substituting $k=\alpha+1$ into (3.3.2) we get

$$
\begin{aligned}
\Delta^{\alpha+1} \epsilon_{\nu} & =A_{n-\nu}^{\alpha} \Delta^{\alpha+1} \frac{1}{\nu}+\sum_{j=1}^{k} C_{\alpha+1, j} A_{n-\nu}^{\alpha-j} \Delta^{\alpha+1-j} \frac{1}{\nu+j} \\
& =A_{n-\nu}^{\alpha} \Delta^{\alpha+1} \frac{1}{\nu}+\sum_{j=1}^{k} A_{n-\nu}^{\alpha-j} O\left(\frac{1}{\nu^{\alpha+2-j}}\right) .
\end{aligned}
$$

Hence 


$$
\begin{aligned}
P_{n} & =\sum_{\nu=1}^{n-\alpha-1} A_{n-\nu}^{\alpha} C_{\nu}^{\alpha+1} \Delta^{\alpha+1} \frac{1}{\nu}+\sum_{j=1}^{\alpha+1} \sum_{\nu=1}^{n-\alpha-1} A_{n-\nu}^{\alpha-j} C_{\nu}^{\alpha+1} O\left(\frac{1}{\nu^{\alpha+2-j}}\right) \\
& =\sum_{\nu=1}^{n-\alpha-1} A_{n-\nu}^{\alpha} C_{\nu}^{\alpha+1} \Delta^{\alpha+1} \frac{1}{\nu}+\sum_{j=1}^{\alpha+1} \sum_{\nu=1}^{n-\alpha-1} A_{n-\nu}^{\alpha-j} O\left(A_{\nu}^{j-1}\right) \\
& =\sum_{\nu=1}^{n-\alpha-1} A_{n-\nu}^{\alpha} C_{\nu}^{\alpha+1} \Delta^{\alpha+1} \frac{1}{\nu}+o\left(n^{\alpha}\right) .
\end{aligned}
$$

We may also write

$$
P_{n}=\sum_{\nu=1}^{n} A_{n-\nu}^{\alpha} C_{\nu}^{\alpha+1} \Delta^{\alpha+1} \frac{1}{\nu}+o\left(n^{\alpha}\right) .
$$

From this and from (3.3.1) and (3.3.3) we get

$$
\sigma_{n}^{\alpha}=\frac{1}{A_{n}^{\alpha}} \sum_{\nu=1}^{n} A_{n-\nu}^{\alpha} C_{\nu}^{\alpha+1} \Delta^{\alpha+1} \frac{1}{\nu}+o(1) .
$$

The first term on the right is the $\alpha$ th Cesàro mean $\tau_{n}^{\alpha}$ of the series

$$
0+\sum_{\nu=1}^{\infty} C_{\nu}^{\alpha+1} \Delta^{\alpha+1} \frac{1}{\nu}
$$

whose terms are $o(1 / \nu)$. By hypothesis, the series (3.2.1) is summable $A$ to 0 , and so on account of (i) summable $A(C, \alpha)$ to 0 . From (3.3.4) it follows that the series (3.3.5) is summable $A(C, \alpha)$ to 0 , and so converges to 0 . Hence $\tau_{n}^{\alpha} \rightarrow 0$, which on account of (3.3.4) gives

$$
\stackrel{\alpha}{\sigma_{n}} \rightarrow 0 \text {. }
$$

Lemma $\mathrm{G}$ is thus established in the case when $\alpha$ is an integer.

In the case of fractional $\alpha>-1$ the proof is similar. We write

$$
\beta=[\alpha]+1, \quad \eta_{0}=0, \quad \eta_{\nu}=\eta_{\nu}^{(n)}=A_{\nu}^{\alpha}\left(\frac{1}{\nu}-\frac{1}{n}\right) \quad \text { for } 0<\nu \leqq n .
$$

Then

$$
s_{n}^{\alpha}=\frac{1}{n} \sum_{\nu=1}^{n} c_{\nu} A_{n-\nu}^{\alpha}+\sum_{\nu=1}^{n} \eta_{\nu} c_{\nu}=o\left(n^{\alpha}\right)+\sum_{\nu=1}^{\beta} \eta_{\nu} c_{\nu}
$$

since the sequence $\left\{c_{\nu}\right\}$ is by hypothesis summable $(C, \alpha+1)$ to 0 . On the other hand,

$$
\sum_{\nu=1}^{n} \eta_{\nu} C_{\nu}=\sum_{\nu=1}^{n-\beta-1} C_{\nu}^{\beta+1} \Delta^{\beta+1} \eta_{\nu}+\sum_{k=0}^{\beta} C_{n-k}^{k+1} \Delta^{k} \eta_{n-k}
$$


Arguing as before (we omit the details, which remain essentially unchanged) we get instead of (3.3.4) the formula

$$
\sigma_{n}^{\alpha}=\frac{1}{A_{n}^{\alpha}} \sum_{\nu=1}^{n} A_{n-\nu}^{\alpha} C_{\nu}^{\beta+1} \Delta^{\beta+1} \frac{1}{\nu}+o(1)
$$

which in the case $\alpha \geqq 0$ gives (3.3.6) (the argument is then the same as in the case of integral $\alpha$ ).

If $-1<\alpha<0$, then summability $(C, \alpha+1)$ of the sequence $\left\{c_{\nu}\right\}$ implies summability $(C, 1)$ of that sequence, to the limit 0 , and so on account of the formula (3.3.4) with $\alpha=0$ we have

$$
s_{n}=\sum_{\nu=1}^{n} C_{\nu}^{1} \Delta \frac{1}{\nu}+o(1) .
$$

The series (3.2.1) being summable $A$, the series $\sum C_{\nu}^{1} \Delta 1 / \nu$, whose terms are $o(1 / \nu)$, is convergent, and so summable $(C, \alpha)$ (cf. (iii)). In other words, the right-hand side of the formula (3.3.7), where $-1<\alpha<0, \beta=0$, tends to 0 . This proves (3.3.6) also in the case $-1<\alpha<0$, and so Lemma $G$ is proved.

3.4. Lemma H. If a series is summable $A$ and its Cesàro means of order $\alpha$ are bounded $(\alpha>-1)$, then the series in summable $(C, \alpha+\epsilon)$ for any $\epsilon>0$.

This lemma is well known $\left({ }^{16}\right)$.

Lemma I. If the Cesàro means $\sigma_{n}^{\alpha}=s_{n}^{\alpha} / A_{n}^{\alpha}$, where $\alpha>-1$, of a series $a_{0}+a_{1}+\cdots$ are bounded below, and if the series is summable $A$, then the series is summable $(C, \alpha+1)$.

In the case $\alpha=0$ the lemma reduces to a very well known theorem of Littlewood. The general result may either be deduced from Littlewood's theorem by a certain comparatively simple argument $\left({ }^{17}\right)$, or may be proved exactly in the same way as the Littlewood theorem. We shall follow the latter course, using the familiar device of $\operatorname{Karamata}\left({ }^{18}\right)$.

Without loss of generality we may assume that the expressions $\sigma_{n}^{\alpha}$ are all positive. By hypothesis,

$$
\sum_{n=0}^{\infty} a_{n} r^{n}=(1-r)^{\alpha+1} \sum_{n=0}^{\infty} s_{n}^{\alpha} r^{n} \rightarrow s \quad \text { as } r \rightarrow 1,
$$

where $s$ is the $A$-sum of the series $a_{0}+a_{1}+\cdots$ Replacing $r$ by $r^{k+1}$, where $k$ is any non-negative integer, we easily obtain from the last relation

(16) See Andersen [1]. A proof may also be found in Zygmund [1].

(17) See Zygmund [2, p. 329].

(18) Karamata [1]. 


$$
(1-r)^{\alpha+1} \sum_{n=0}^{\infty} s_{n}^{\alpha} r^{(k+1) n} \rightarrow \frac{s}{(k+1)^{\alpha+1}}=\frac{s}{\Gamma(\alpha+1)} \int_{0}^{1} x^{k}\left(\log \frac{1}{x}\right)^{\alpha} d x,
$$

and so, if $P(x)$ is any polynomial,

$$
(1-r)^{\alpha+1} \sum_{n=0}^{\infty} s_{n}^{\alpha} r^{n} P\left(r^{n}\right) \rightarrow \frac{s}{\Gamma(\alpha+1)} \int_{0}^{1} P(x)\left(\log \frac{1}{x}\right)^{\alpha} d x
$$

Let $Q(x)$ be any function defined and bounded in the interval $0 \leqq x \leqq 1$. Let us assume furthermore that $Q(x)$ is continuous except at some point where it has a jump. Approximating $Q(x)$ above and below by polynomials and taking account of the positiveness of the expressions $s_{n}^{\alpha}$, we deduce from (3.4.1) that

$$
(1-r)^{\alpha+1} \sum_{n=0}^{\infty} s_{n}^{\alpha} r^{n} Q\left(r^{n}\right) \rightarrow \frac{s}{\Gamma(\alpha+1)} \int_{0}^{1} Q(x)\left(\log \frac{1}{x}\right)^{\alpha} d x .
$$

Let us define $Q(x)$ by the conditions

$$
Q(x)=0 \quad \text { for } \quad 0 \leqq x<e^{-1}, \quad Q(x)=\frac{1}{x} \quad \text { for } \quad e^{-1} \leqq x \leqq 1 .
$$

The right-hand side of (3.4.2) is then equal to $s / \Gamma(\alpha+2)$. Hence, if we set $r=e^{-1 / N}$, and make $N$ tend to $+\infty$, the relation (3.4.2) gives

$$
\frac{1}{N^{\alpha+1}} \sum_{n=0}^{N} s_{n}^{\alpha} \rightarrow \frac{s}{\Gamma(\alpha+2)},
$$

or, since $A_{n}^{\alpha+1} \simeq n^{\alpha+1} / \Gamma(\alpha+2)$,

$$
\frac{s_{N}^{\alpha+1}}{A_{N}^{\alpha+1}} \rightarrow s
$$

This completes the proof of the lemma.

3.5. Let $\sigma_{n}^{\alpha}(\theta)$ denote the Cesàro means of the trigonometric series

$$
\frac{1}{2} a_{0}+\sum_{n=1}^{\infty}\left(a_{n} \cos n \theta+b_{n} \sin n \theta\right) .
$$

The series will be said to be $f i n i t e\left(C^{*}, \alpha\right)$ at a point $\theta_{0}$, if for any $A>0$ there is a number $B=B(A)$, such that

$$
\left|\sigma_{n}^{\alpha}\left(\theta_{0}+h\right)\right| \leqq B \text { for }|h| \leqq \frac{A}{n} \text { and } n=1,2, \cdots .
$$

Similarly the series (3.5.1) will be said to be finite $A^{*}$ at the point $\theta_{0}$, if for any $C>0$ the harmonic function 


$$
\frac{1}{2} a_{0}+\sum_{n=1}^{\infty}\left(a_{n} \cos n \theta+b_{n} \sin n \theta\right) r^{n}
$$

is bounded in the neighborhood of the point $e^{i \theta_{0}}$ satisfying the inequality $\left|r e^{i \theta}-e^{i \theta_{0}}\right| \leqq C(1-r)$. It is plain that Lemmas $\mathrm{D}, \mathrm{E}, \mathrm{F}, \mathrm{G}$ have the following analogues.

Lemma $D_{1}$. A necessary and sufficient condition that the series (3.5.1) should be finite $\left(C^{*}, \alpha\right), \alpha>-1$, at the point $\theta_{0}$, is that

(i) the series (3.5.1) should be finite $(C, \alpha)$ at the point $\theta_{0}$; and that

(ii) there should exist a sequence $\left\{h_{n}\right\}$ satisfying the condition

$$
0<\delta \leqq\left|n h_{n}\right| \leqq \pi-\delta, \quad \delta \text { independent of } n,
$$

and such that

$$
\sigma_{\nu}^{\alpha}\left(\theta_{0}+h_{n}\right)=O(1) \quad \text { for } \nu \rightarrow \infty, \nu \leqq n .
$$

Lемма $\mathrm{E}_{1}$. If the series (3.5.1) is finite $\left(C^{*}, \alpha\right), \alpha>-1$, at a point $\theta_{0}$, then the sequence $n\left(b_{n} \cos n \theta_{0}-a_{n} \sin n \theta_{0}\right)$ is finite $(C, \alpha+1)$.

Lemma $F_{1}$. If the series (3.5.1) is finite $\left(C^{*}, \alpha\right), \alpha>-1$, at the point $\theta_{0}$, it is also finite $A^{*}$ at that point.

Lемма $G_{1}$. If the series $a_{0}+a_{1}+\cdots$ is finite $A$, and if the sequence $\left\{\nu a_{\nu}\right\}$ is finite $(C, \alpha+1)$, then the series $a_{0}+a_{1}+\cdots$ is finite $(C, \alpha)(\alpha>-1)$.

\section{Proofs of fundamental theorems}

4.1. LeMmA J. If $G$ is any measurable set of positive measure and $\theta_{0}$ a point of density of $G$, then for any $\lambda>0$ there is a sequence of numbers $\left\{\alpha_{n}\right\}$ such that

$$
\begin{aligned}
& n \alpha_{n} \rightarrow \lambda, \\
& \theta_{0} \pm \alpha_{n} \text { belongs to } G \text { for all } n>n_{0} .
\end{aligned}
$$

Proof. Since $\theta_{0}$ is a point of density, the average densities of the set $G$ in the intervals

$$
\begin{array}{r}
\left(\theta_{0}+\frac{\lambda-\epsilon}{n}, \theta_{0}+\frac{\lambda+\epsilon}{n}\right),\left(\theta_{0}-\frac{\lambda+\epsilon}{n}, \theta_{0}-\frac{\lambda-\epsilon}{n}\right), \\
\text { where } 0<\epsilon<\lambda,
\end{array}
$$

tend to 1 as $n$ tends to infinity. It follows that for $n$ large enough these average densities exceed $\frac{1}{2}$, and so there is a number $\beta_{n}$ such that

$$
\frac{\lambda-\epsilon}{n} \leqq \beta_{n} \leqq \frac{\lambda+\epsilon}{n}, \quad \theta_{0} \pm \beta_{n} \in G, \quad n>n_{0} .
$$


Thence it is not difficult to deduce the existence of a sequence $\left\{\alpha_{n}\right\}$ satisfying the conditions (4.1.1).

Lемма K. If the series

$$
\frac{1}{2} a_{0}+\sum_{n=1}^{\infty}\left(a_{n} \cos n \theta+b_{n} \sin n \theta\right)
$$

is summable $(C, \alpha), \alpha>-1$, for $\theta \in E$, then the series is summable $\left(C^{*}, \alpha\right)$ almost everywhere in $E\left({ }^{19}\right)$.

Similarly, if the series (4.1.2) is finite $(C, \alpha)$ at every point of $E$, the series is finite $\left(C^{*}, \alpha\right)$ almost everywhere in $E$.

Proof. Let us suppose that the series (4.1.2) is summable $(C, \alpha)$ for $\theta \in E$, and let $s(\theta)$ denote the $(C, \alpha)$-sum of the series. Let $G$ be any subset of positive measure of $E$, such that the Cesàro means $\sigma_{n}^{\alpha}(\theta)$ of the series (4.1.2) tend to $s(\theta)$ uniformly on $G$. In particular, $s(\theta)$ is continuous on $G$. Let $\theta_{0} \in G$ be a point of density of $G$, and let $\left\{\alpha_{n}\right\}$ be any sequence of numbers such that

$$
\frac{1}{4} \pi \leqq n \alpha_{n} \leqq \frac{3}{4} \pi, \quad \theta_{0}+\alpha_{n} \in G
$$

for all $n>n_{0}$. Since

$$
\sigma_{\nu}^{\alpha}\left(\theta_{0}+\alpha_{n}\right)-s\left(\theta_{0}+\alpha_{n}\right) \rightarrow 0 \quad \text { for } \nu \rightarrow \infty, n \geqq \nu,
$$

and since $s\left(\theta_{0}+\alpha_{n}\right) \rightarrow s\left(\theta_{0}\right)$, it follows that $\sigma_{\nu}^{\alpha}\left(\theta_{0}+\alpha_{n}\right) \rightarrow s\left(\theta_{0}\right)$ for $\nu \rightarrow \infty, n \geqq \nu$. An application of Lemma $D$ shows that the series (4.1.2) is summable $\left(C^{*}, \alpha\right)$ at the point $\theta_{0}$, that is, is summable $\left(C^{*}, \alpha\right)$ almost everywhere in $G$.

Since the measure of $E-G$ may be arbitrarily small, this proves the first part of the lemma. The second part may be proved in a similar way.

4.2. LemMA L. Let

$$
F(z)=u(r, \theta)+i v(r, \theta), \quad z=r e^{i \theta},
$$

be a function regular inside the unit circle, and let us suppose that there is a set $E$ of positive measure situated on the circumference $|z|=1$ and such that for any $z_{0} \in E$ the function $u(r, \theta)$ is bounded when $z$ tends to $z_{0}$ along non-tangential paths. Under these conditions for almost every point $z_{0} \in E$ the functions $u(r, \theta)$ and $v(r, \theta)$ tend to finite limits when $z$ tends to $z_{0}$ along any non-tangential paths.

This lemma is known $\left({ }^{20}\right)$. It plays an essential part in our proofs of Theorems 1 and 2. It may also be stated in the following form: If a trigonometric series is finite $A^{*}$ at every point of a set $E$ of positive measure, then the series itself and its conjugate are summable $A^{*}$ almost everywhere in $E$.

(19) See Plessner [1] for $\alpha=0,1,2, \cdots$.

$\left.{ }^{(20}\right)$ Privaloff [1]. A more general result, obtained by an argument similar to Privaloff's, will be found in Plessner [2]. 
4.3. From the preceding results we may deduce without difficulty the following special case of Theorem 1:

Lemma M. If a trigonometric series is summable $(C, \beta), \beta>-1$, at every point of a set $E$, the conjugate series is summable $(C, \beta)$ almost everywhere in $E\left({ }^{21}\right)$.

Proof. If the series (4.1.2) is summable $(C, \beta)$ in $E$, then it is summable $\left(C^{*}, \beta\right)$ almost everywhere in $E$ (Lemma $\mathrm{K}$ ). It follows (Lemma $\mathrm{E}$ ) that

$$
(C, \beta+1) n\left(b_{n} \cos n \theta-a_{n} \sin n \theta\right) \rightarrow 0 \quad \text { at almost every point of } E .
$$

On the other hand, from Lemmas $\mathrm{F}$ and $\mathrm{L}$ it follows that the conjugate series

$$
\sum_{n=1}^{\infty}\left(a_{n} \sin n \theta-b_{n} \cos n \theta\right)
$$

is summable $A^{*}$, and so also $A$, almost everywhere in $E$. From this and from (4.3.1) we see (cf. Lemma $G$ ) that the series (4.3.2) is summable $(C, \beta)$ almost everywhere in $E$.

4.4. Lemma N. If at every point $\theta$ of a set $E$ of positive measure the series

$$
\frac{1}{2} a_{0}+\sum_{n=1}^{\infty}\left(a_{n} \cos n \theta+b_{n} \sin n \theta\right)
$$

is summable $(C, \alpha+1)$, where $\alpha>-1$, to sum $s(\theta)$, and if

$$
\sigma_{\alpha}(\theta)>-\infty
$$

for $\theta \in E$,

then

$$
\begin{gathered}
\sigma^{\alpha}(\theta)<+\infty, \\
s(\theta)=\frac{1}{2}\left\{\sigma_{\alpha}(\theta)+\sigma^{\alpha}(\theta)\right\}
\end{gathered}
$$

at almost every point of $E$.

Proof $\left({ }^{22}\right)$. Let us suppose first that $\alpha$ is an integer. From (4.4.2) and from Egoroff's well known theorem we deduce that there is a subset $G$ of $E$, of measure differing as little as we please from that of $E$ and such that

$$
\sigma_{n}^{\alpha}(\theta)>\sigma_{\alpha}(\theta)-\epsilon_{n}
$$

for $\theta \in G$,

where $\epsilon_{1}, \epsilon_{2}, \cdots$ is a sequence of numbers independent of $\theta$ and tending to 0 . The functions $s(\theta)$ and $\sigma_{\alpha}(\theta)$ are measurable, and so if we remove from $E$ subsets of arbitrarily small measure, these functions will be continuous on the

(21) See Plessner [1] (for the case $\alpha=0,1, \cdots$ ), Marcinkiewicz and Zygmund [1].

(22) Lemmas $\mathrm{N}$ and $\mathrm{O}$ of this paper are taken without essential changes from Marcinkiewicz and Zygmund [1]. 
remaining set. Without loss of generality we may assume that the functions $s(\theta)$ and $\sigma_{\alpha}(\theta)$ are continuous on $G$.

Let $\theta_{0}$ be any point of density of the set $G$ and belonging to $G$, and let $\left\{\beta_{n}\right\}$ be a sequence of numbers satisfying the conditions $n \beta_{n} \rightarrow \pi, \theta_{0} \pm \beta_{n} \in G$ (cf. Lemma J). It follows from (4.4.5) that

$$
\frac{1}{2}\left\{\sigma_{n}^{\alpha}\left(\theta_{0}+\beta_{n}\right)+\sigma_{n}^{\alpha}\left(\theta_{0}-\beta_{n}\right)\right\}>\frac{1}{2}\left\{\sigma_{\alpha}\left(\theta_{0}+\beta_{n}\right)+\sigma_{\alpha}\left(\theta_{0}-\beta_{n}\right)\right\}-\epsilon_{n} .
$$

If we introduce the notation

$$
\begin{array}{crr}
C_{0}(\theta)=\frac{1}{2} a_{0}, \quad C_{\nu}(\theta)=a_{\nu} \cos \nu \theta+b_{\nu} \sin \nu \theta & \text { for } \nu \geqq 0, \\
\lambda_{\nu}=\lambda_{\nu}^{(n)}=A_{n-\nu}^{\alpha} \cos \nu \beta_{n} & \text { for } 0 \leqq \nu \leqq n,
\end{array}
$$

the left-hand side of the last inequality may be written

$$
\begin{aligned}
\frac{1}{2}\left\{\sigma_{n}^{\alpha}\left(\theta_{0}+\beta_{n}\right)+\sigma_{n}^{\alpha}\left(\theta_{0}-\beta_{n}\right)\right\} & =\frac{1}{A_{n}^{\alpha}} \sum_{\nu=0}^{n} A_{n-\nu}^{\alpha} C_{\nu}\left(\theta_{0}\right) \cos \nu \beta_{n} \\
& =\frac{1}{A_{n}^{\alpha}} \sum_{\nu=0}^{n} C_{\nu}\left(\theta_{0}\right) \lambda_{\nu} .
\end{aligned}
$$

Let $s_{n}^{k}(\theta)$ denote the $k$ th Cesàro sums of the series (4.4.1). Applying summation by parts $\alpha+2$ times, we may represent the last sum in the form

$$
\frac{1}{A_{n}^{\alpha}} \sum_{\nu=0}^{n-\alpha-2} s_{\nu}^{\alpha+1}\left(\theta_{0}\right) \Delta^{\alpha+2} \lambda_{\nu}+\frac{1}{A_{n}^{\alpha}} \sum_{j=0}^{\alpha+1} s_{n-j}^{j}\left(\theta_{0}\right) \Delta^{j} \lambda_{n-j}=P_{n}+Q_{n},
$$

say. Since $A_{\mu}^{-1}=A_{\mu}^{-2}=0$ for $\mu>0$, we may write

$$
\begin{aligned}
\Delta^{\alpha+2} \lambda_{\nu} & =\sum_{j=0}^{\alpha+2} C_{\alpha+2, j} A_{n-\nu}^{\alpha-j} \Delta^{\alpha+2-j} \cos (\nu+j) \beta_{n}=\sum_{j=0}^{\alpha} C_{\alpha+2, j} A_{n-\nu}^{\alpha-j} \Delta^{\alpha+2-j} \cos (\nu+j) \beta_{n} \\
& =\sum_{j=0}^{\alpha} A_{n-\nu}^{\alpha-j} O\left(\beta_{n}^{j-\alpha-2}\right)=O\left(n^{-2}\right)
\end{aligned}
$$

for $0 \leqq \nu \leqq n-\alpha-2$. Assuming for simplicity that $s\left(\theta_{0}\right)=0$, that is, that

$$
s_{n}^{\alpha+1}\left(\theta_{0}\right)=o\left(n^{\alpha+1}\right),
$$

we see that $P_{n}$ is equal to a sum of $\alpha+1$ expressions of the form

so that

$$
o\left(\frac{1}{n^{2} A_{n}^{\alpha}}\right) \sum_{\nu=0}^{n-\alpha-2} o\left(A_{\nu}^{\alpha+1}\right)=o(1)
$$

$$
P_{n}=o(1) \text {. }
$$

Since 


$$
\Delta^{j} \lambda_{\nu}=\sum_{h=0}^{j} C_{j, h} A_{n-\nu}^{\alpha-h} \Delta^{j-h} \cos (\nu+h) \beta_{n} \quad \text { for } 0 \leqq \nu \leqq n-j,
$$

the coefficient of $s_{n-j}^{j}\left(\theta_{0}\right)$ in $A_{n}^{\alpha} Q_{n}$ is equal to

$$
\begin{aligned}
A_{j}^{\alpha-j} \cos n \beta_{n}+\sum_{h=0}^{j-1} C_{j, h} A_{j}^{\alpha-h} \Delta^{j-h} \cos (n-j+h) \beta_{n} \\
=A_{j}^{\alpha-j} \cos n \beta_{n}+\sum_{h=0}^{j-1} O\left(n^{h-j}\right)=A_{j}^{\alpha-j} \cos n \beta_{n}+O\left(n^{-1}\right)
\end{aligned}
$$

for $j=0,1, \cdots, \alpha+1$. On the other hand, the condition $s_{m}^{\alpha+1}\left(\theta_{0}\right)=o\left(m^{\alpha+1}\right) \mathrm{im}$ plies

Hence,

$$
s_{m}^{j}\left(\theta_{0}\right)=o\left(m^{\alpha+1}\right) \quad \text { for } j=0,1, \cdots, \alpha .
$$

$$
Q_{n}=\frac{\cos n \beta_{n}}{A_{n}^{\alpha}} \sum_{j=0}^{\alpha+1} s_{n-j}^{j}\left(\theta_{0}\right) A_{j}^{\alpha-j}+o(1)=\frac{\cos n \beta_{n}}{A_{n}^{\alpha}} \sum_{j=0}^{\alpha} s_{n-j}^{j}\left(\theta_{0}\right) A_{j}^{\alpha-j}+o(1)
$$

(since $A_{j}^{-1}=0$ for $j>0$ ).

The left-hand side of the inequality (4.4.6) is equal to $P_{n}+Q_{n}$, and so on account of (4.4.8) we get

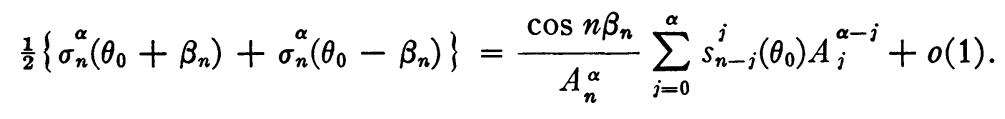

The function $\sigma_{\alpha}(\theta)$ is by hypothesis continuous at the point $\theta_{0}$ with respect to the set $G$. The right-hand side of the inequality (4.4.6) tends therefore to $\sigma_{\alpha}\left(\theta_{0}\right)$. From this and from (4.4.9) we deduce that

$$
\liminf _{n \rightarrow \infty} \frac{\cos n \beta_{n}}{A_{n}^{\alpha}} \sum_{j=0}^{\alpha} s_{n-j}^{j}\left(\theta_{0}\right) A_{j}^{\alpha-j} \geqq \sigma_{\alpha}\left(\theta_{0}\right)
$$

or, since $n \beta_{n} \rightarrow \pi$,

$$
\limsup _{n \rightarrow \infty} \frac{1}{A_{n}^{\alpha}} \sum_{j=0}^{\alpha} s_{n-j}^{j}\left(\theta_{0}\right) A_{j}^{\alpha-j} \leqq-\sigma_{\alpha}\left(\theta_{0}\right)
$$

4.5. Let us now consider the difference

$$
\begin{aligned}
\sigma_{n}^{\alpha}\left(\theta_{0}\right)-\sigma_{n}^{\alpha+1}\left(\theta_{0}\right) & =\sum_{\nu=0}^{n} C_{\nu}\left(\theta_{0}\right)\left\{\frac{A_{n-\nu}^{\alpha}}{A_{n}^{\alpha}}-\frac{A_{n-\nu}^{\alpha+1}}{A_{n}^{\alpha+1}}\right\} \\
& =\frac{1}{(n+\alpha+1) A_{n}^{\alpha}} \sum_{\nu=0}^{n} \nu A_{n-\nu}^{\alpha} C_{\nu}\left(\theta_{0}\right) .
\end{aligned}
$$

The last expression is analogous to (4.4.7), except that the factor $\cos \nu \beta_{n}$ is replaced by 


$$
\gamma_{\nu}=\gamma_{\nu}^{(n)}=\frac{\nu}{n+\alpha+1}
$$

which for our purposes possesses the same properties as $\cos \nu \beta_{n}$, namely

$$
\Delta^{k} \gamma_{\nu}=O\left(n^{-k}\right), \quad \quad k=0,1, \cdots
$$

(as a matter of fact, $\Delta^{k} \gamma_{\nu}=0$ for $k>1$ ). Hence, repeating the argument which gave (4.4.9), we get

$$
\sigma_{n}^{\alpha}\left(\theta_{0}\right)-\sigma_{n}^{\alpha+1}\left(\theta_{0}\right)=\frac{n}{n+\alpha+1} \frac{1}{A_{n}^{\alpha}} \sum_{j=0}^{\alpha} s_{n-j}^{j}\left(\theta_{0}\right) A_{j}^{\alpha-j}+o(1) .
$$

From (4.5.3) and from the inequality

$$
\limsup _{n \rightarrow \infty} \frac{1}{A_{n}^{\alpha}} \sum_{j=0}^{\alpha} s_{n-j}^{j}\left(\theta_{0}\right) A_{j}^{\alpha-j} \leqq-\sigma_{\alpha}\left(\theta_{0}\right),
$$

we deduce that

$$
\limsup _{n \rightarrow \infty}\left\{\sigma_{n}^{\alpha}\left(\theta_{0}\right)-\sigma_{n}^{\alpha+1}\left(\theta_{0}\right)\right\} \leqq-\sigma_{\alpha}\left(\theta_{0}\right)
$$

or that

$$
\sigma_{\alpha}\left(\theta_{0}\right)+\sigma^{\alpha}\left(\theta_{0}\right) \leqq 0 .
$$

This inequality was established under the hypothesis $\sigma_{n}^{\alpha+1}\left(\theta_{0}\right) \rightarrow 0$. In the general case, which may be reduced to the former by subtracting the constant $s\left(\theta_{0}\right)$ from the series (4.4.1), we obtain

$$
\sigma_{\alpha}\left(\theta_{0}\right)+\sigma^{\alpha}\left(\theta_{0}\right) \leqq 2 s\left(\theta_{0}\right) .
$$

This relation holds almost everywhere in $G$, and so almost everywhere in $E$. Hence (4.4.3) is true at almost every point of $E$.

Let us now change the signs of all the coefficients of the series (4.4.1) and let us apply the inequality (4.5.1) to the new series. We get that

$$
\sigma_{\alpha}\left(\theta_{0}\right)+\sigma^{\alpha}\left(\theta_{0}\right) \geqq 2 s\left(\theta_{0}\right)
$$

almost everywhere in $E$, which on account of (4.5.1) gives (4.4.4) at almost every point of $E$. Thus Lemma $\mathrm{N}$ is proved in the case of integral $\alpha$.

4.6. Passing to the case of fractional $\alpha>-1$, we set

$$
\beta=[\alpha]+1,
$$

so that $\alpha<\beta<\alpha+1$. Let us assume that the series (4.4.1) is summable not only $(C, \alpha+1)$, but also $(C, \beta)$ on $E$. Let us define the set $G$ in the same way as before, and let $\theta_{0} \in G$ be any point of density of $G$. Let us assume for simplicity that

$$
\stackrel{\beta}{\sigma_{n}}\left(\theta_{0}\right) \rightarrow 0 .
$$


Applying summation by parts $\beta+1$ times to the right-hand side of the equation

$$
\frac{1}{2}\left\{\sigma_{n}^{\alpha}\left(\theta_{0}+\beta_{n}\right)+\sigma_{n}^{\alpha}\left(\theta_{0}-\beta_{n}\right)\right\}=\frac{1}{A_{n}^{\alpha}} \sum_{\nu=0}^{n} C_{\nu}\left(\theta_{0}\right) \lambda_{\nu}
$$

(cf. (4.4.7)) where $n \beta_{n} \rightarrow \pi, \theta_{0} \pm \beta_{n} \in G$, we get the expression

$$
\frac{1}{A_{n}^{\alpha}} \sum_{\nu=0}^{n-\beta-1} s_{\nu}^{\beta}\left(\theta_{0}\right) \Delta^{\beta+1} \lambda_{\nu}+\frac{1}{A_{n}^{\alpha}} \sum_{j=0}^{\beta} s_{n-j}^{j}\left(\theta_{0}\right) \Delta^{j} \lambda_{n-j}=P_{n}+Q_{n},
$$

say. To estimate $P_{n}$ we use the formula

$$
\begin{array}{r}
\Delta^{\beta+1} \lambda_{\nu}=\sum_{j=0}^{\beta} C_{\beta+1, j} A_{n-\nu}^{\alpha-j} \Delta^{\beta+1-j} \cos (\nu+j) \beta_{n}+A_{n-\nu}^{\alpha-\beta-1} \cos (\nu+\beta+1) \beta_{n} \\
\text { for } 0 \leqq \nu \leqq n-\beta-1 .
\end{array}
$$

Correspondingly,

$$
\begin{aligned}
P_{n} & =\sum_{j=0}^{\beta} \frac{O\left(n^{j-\beta-1}\right)}{A_{n}^{\alpha}} \sum_{\nu=0}^{n-\beta-1} o\left(A_{n}^{\beta}\right) A_{n-\nu}^{\alpha-j}+\frac{1}{A_{n}^{\alpha}} \sum_{\nu=0}^{n-\beta-1} s_{\nu}^{\beta}\left(\theta_{0}\right) A_{n-\nu}^{\alpha-\beta-1} \cos (\nu+\beta+1) \beta_{n} \\
& =o(1)+\frac{1}{A_{n}^{\alpha}} \sum_{\nu=0}^{n-\beta-1} s_{\nu}^{\beta}\left(\theta_{0}\right) A_{n-\nu}^{\alpha-\beta-1} \cos (\nu+\beta+1) \beta_{n} .
\end{aligned}
$$

On the other hand, the last expression is equal to

$$
\begin{aligned}
\frac{\cos n \beta_{n}}{A_{n}^{\alpha}} \sum_{\nu=0}^{n-\beta-1} s_{\nu}^{\beta}\left(\theta_{0}\right) A_{n-\nu}^{\alpha-\beta-1} & +\frac{1}{A_{n}^{\alpha}} \sum_{\nu=0}^{n-\beta-1} o\left(A_{\nu}^{\beta}\right)\left|A_{n-\nu}^{\alpha-\beta-1}\right| O(n-\nu) \beta_{n} \\
= & \frac{\cos n \beta_{n}}{A_{n}^{\alpha}} \sum_{\nu=0}^{n-\beta-1} s_{\nu}^{\beta}\left(\theta_{0}\right) A_{n-\nu}^{\alpha-\beta-1}+\frac{\beta_{n}}{A_{n}^{\alpha}} \sum_{\nu=0}^{n-\beta-1} o\left(A_{\nu}^{\beta}\right) O\left(A_{n-\nu}^{\alpha-\beta}\right) \\
= & \frac{\cos n \beta_{n}}{A_{n}^{\alpha}} \sum_{\nu=0}^{n-\beta-1} s_{\nu}^{\beta}\left(\theta_{0}\right) A_{n-\nu}^{\alpha-\beta-1}+o(1),
\end{aligned}
$$

so that

$$
P_{n}=\frac{\cos n \beta_{n}}{A_{n}^{\alpha}} \sum_{\nu=0}^{n-\beta-1} s_{\nu}^{\beta}\left(\theta_{0}\right) A_{n-\nu}^{\alpha-\beta-1}+o(1) .
$$

Furthermore, from the formula

we get

$$
\Delta^{j} \lambda_{\nu}=\sum_{h=0}^{j} C_{j, h} A_{n-\nu}^{\alpha-j} \Delta^{j-h} \cos (\nu+h) \beta_{n}
$$




$$
\begin{aligned}
Q_{n} & =\frac{1}{A_{n}^{\alpha}} \sum_{j=0}^{\beta} s_{n-j}^{j}\left(\theta_{0}\right) \sum_{h=0}^{j-1} C_{j, h} A_{j}^{\alpha-j} O\left(\beta_{n}^{j-h}\right)+\frac{1}{A_{n}^{\alpha}} \sum_{j=0}^{\beta} s_{n-j}^{j}\left(\theta_{0}\right) A_{j}^{\alpha-j} \cos n \beta_{n} \\
& =\frac{1}{A_{n}^{\alpha}} \sum_{j=0}^{\beta} o\left(n^{\beta}\right) O\left(n^{-1}\right)+\frac{\cos n \beta_{n}}{A_{n}^{\alpha}} \sum_{j=0}^{\beta} s_{n-j}^{j}\left(\theta_{0}\right) A_{j}^{\alpha-j} \\
& =o(1)+\frac{\cos n \beta_{n}}{A_{n}^{\alpha}} \sum_{j=0}^{\beta} s_{n-j}^{j}\left(\theta_{0}\right) A_{j}^{\alpha-j} .
\end{aligned}
$$

Hence the expression (4.6.1) is equal to

$$
\frac{\cos n \beta_{n}}{A_{n}^{\alpha}}\left\{\sum_{\nu=0}^{n-\beta-1} s_{\nu}^{\beta}\left(\theta_{0}\right) A_{n-\nu}^{\alpha-\beta-1}+\sum_{j=0}^{\beta} s_{n-j}^{j}\left(\theta_{0}\right) A_{j}^{\alpha-j}\right\}+o(1) .
$$

Since $n \beta_{n} \rightarrow \pi, \theta_{0} \pm \beta_{n} \in G$, it follows that

$$
\limsup _{n \rightarrow \infty} \frac{1}{A_{n}^{\alpha}}\left\{\sum_{\nu=0}^{n-\beta-1} s_{\nu}^{\beta}\left(\theta_{0}\right) A_{n-\nu}^{\alpha-\beta-1}+\sum_{j=0}^{\beta} s_{n-j}^{j}\left(\theta_{0}\right) A_{j}^{\alpha-j}\right\} \leqq-\sigma_{\alpha}\left(\theta_{0}\right) .
$$

Let us now consider the equation (4.5.1). The argument which transformed (4.6.1) into (4.6.2) gives

$\sigma_{n}^{\alpha}\left(\theta_{0}\right)-\sigma_{n}^{\alpha+1}\left(\theta_{0}\right)$

$$
=\frac{n}{n+\alpha+1} \frac{1}{A_{n}^{\alpha}}\left\{\sum_{\nu=0}^{n-\beta-1} s_{\nu}^{\beta}\left(\theta_{0}\right) A_{n-\nu}^{\alpha-\beta-1}+\sum_{j=0}^{\beta} s_{n-j}^{j}\left(\theta_{0}\right) A_{j}^{\alpha-j}\right\}+o(1) .
$$

Comparing this with (4.6.3) we get

$$
\underset{n \rightarrow \infty}{\limsup }\left\{\sigma_{n}^{\alpha}\left(\theta_{0}\right)-\sigma_{n}^{\alpha+1}\left(\theta_{0}\right)\right\} \leqq-\sigma_{\alpha}\left(\theta_{0}\right),
$$

or

$$
\sigma^{\alpha}\left(\theta_{0}\right)+\sigma_{\alpha}\left(\theta_{0}\right) \leqq 0 .
$$

If $s\left(\theta_{0}\right) \neq 0$, this inequality is to be replaced by

$$
\sigma^{\alpha}\left(\theta_{0}\right)+\sigma_{\alpha}\left(\theta_{0}\right) \leqq 2 s\left(\theta_{0}\right) \text {. }
$$

This gives $\sigma^{\alpha}\left(\theta_{0}\right)<+\infty$, and so (4.4.3) is true almost everywhere in $E$. Hence applying (4.6.5) (with $\theta_{0}$ replaced by general $\theta$ ) to the series obtained from (4.4.1) by changing the signs of the coefficients of the latter series, we obtain

$$
\sigma^{\alpha}(\theta)+\sigma_{\alpha}(\theta) \geqq 2 s(\theta)
$$

and so (4.4.4) is true almost everywhere in $E$.

4.7. We have therefore proved Lemma $\mathrm{N}$ also in the case of fractional $\alpha$, but under an additional assumption, namely that the series (4.4.1) is sum- 
mable not only $(C, \alpha+1)$, but also $(C, \beta)$ in $E$. To remove this assumption we may argue as follows.

First of all, the argument of the preceding section shows that if

$$
\sigma_{\alpha}(\theta)>-\infty, \quad \sigma_{n}^{\beta}(\theta)=O(1)
$$

for $\theta \in E$, then $\sigma^{\alpha}(\theta)<+\infty$, that is,

$$
\underset{\sigma_{n}^{\alpha}}{\alpha}(\theta)=O(1)
$$

almost everywhere in $E$. On the other hand, since $\beta>\alpha$, the hypotheses of Lemma $\mathrm{N}$ imply that

$$
\sigma_{\beta}(\theta)>-\infty, \quad \sigma_{n}^{\beta+1}(\theta) \rightarrow s(\theta) \text { on } E,
$$

and applying Lemma $\mathrm{N}$ in the case of integral $\alpha$ (now $\beta$ ) we get that

$$
\sigma_{n}^{\beta}(\theta)=O(1) \quad \text { almost everywhere in } E .
$$

It follows that under the conditions of Lemma $\mathrm{N}$ (in the case of fractional $\alpha$ ) the relations (4.7.1) are true at almost every point of $E$, so that also (4.7.2) holds almost everywhere in $E$. Now, on account of Lemma $H$, the series (4.4.1) is summable $(C, \beta)$ at every point $\theta$ at which

$$
\sigma_{n}^{\alpha}(\theta)=O(1), \quad \sigma_{n}^{\alpha+1}(\theta) \rightarrow s(\theta) .
$$

Hence, under the conditions of Lemma $\mathrm{N}$, the series (4.4.1) is summable $(C, \beta)$ almost everywhere in $E$. This completes the proof of Lemma $\mathrm{N}$ in the case of fractional $\alpha$.

\subsection{Lемma O. Suppose that for some $\alpha>-1$ the series}

$$
\frac{1}{2} a_{0}+\sum_{\nu=1}^{\infty}\left(a_{\nu} \cos \nu \theta+b_{\nu} \sin \nu \theta\right)
$$

satisfies at every point of a set $E$ of positive measure the condition

$$
-\infty<\sigma_{\alpha}(\theta) \leqq \sigma^{\alpha}(\theta)<+\infty,
$$

and that the conjugate series

$$
\sum_{\nu=1}^{\infty}\left(a_{\nu} \sin \nu \theta-b_{\nu} \cos \nu \theta\right)
$$

is summable $(C, \alpha+1)$ in $E$. Then at almost every point of $E$ the following conditions are satisfied:

$$
\begin{aligned}
-\infty<\tilde{\sigma}_{\alpha}(\theta) & \leqq \tilde{\sigma}^{\alpha}(\theta)<+\infty, \\
\tilde{s}(\theta) & =\frac{1}{2}\left\{\tilde{\sigma}_{\alpha}(\theta)+\tilde{\sigma}^{\alpha}(\theta)\right\},
\end{aligned}
$$




$$
\tilde{\sigma}^{\alpha}(\theta)-\tilde{\sigma}_{\alpha}(\theta)=\sigma^{\alpha}(\theta)-\sigma_{\alpha}(\theta) .
$$

Proof. The argument is so similar to that of Lemma $\mathrm{N}$ that we may condense some parts. Let $G$ be a subset of $E$ such that the functions $\sigma_{\alpha}(\theta)$ and $\sigma^{\alpha}(\theta)$ are continuous on $G$, and that

$$
\sigma_{\alpha}(\theta)-\epsilon_{n} \leqq \sigma_{n}^{\alpha}(\theta) \leqq \sigma_{n}^{\alpha}(\theta)+\epsilon_{n} \quad \text { on } G,
$$

where $\left\{\epsilon_{n}\right\}$ is a sequence of numbers tending to 0 and independent of $\theta$. The measure of $G$ may be as near to that of $E$ as we please. Let $\theta_{0}$ be any point of density of $G$ belonging to $G$, and let $\beta_{n}$ be a sequence of numbers satisfying the conditions

$$
n \beta_{n} \rightarrow \frac{1}{2} \pi \quad \theta_{0} \pm \beta_{n} \in G
$$

Using the notation

$$
\tilde{C}_{0}(\theta)=0, \quad \tilde{C}_{\nu}(\theta)=a_{\nu} \sin \nu \theta-b_{\nu} \cos \nu \theta \quad \text { for } \nu>0
$$

we have the formula

$$
\frac{1}{2}\left\{\sigma_{n}^{\alpha}\left(\theta_{0}+\beta_{n}\right)-\sigma_{n}^{\alpha}\left(\theta_{0}-\beta_{n}\right)\right\}=-\frac{1}{A_{n}^{\alpha}} \sum_{\nu=0}^{n} \tilde{C}_{\nu}\left(\theta_{0}\right) A_{n-\nu}^{\alpha} \sin \nu \beta_{n} .
$$

Here the right-hand side is analogous to the expression (4.4.7), with $C_{\nu}\left(\theta_{0}\right)$ replaced by $\widetilde{C}_{\nu}\left(\theta_{0}\right)$, and $\cos \nu \beta_{n}$ by $-\sin \nu \beta_{n}$. Let $\tilde{s}_{\nu}^{j}(\theta)$ denote the Cesàro sums of the series (4.8.3), and let us assume first that $\tilde{s}\left(\theta_{0}\right)=0$.

In the case of integral $\alpha$ we get from (4.8.8) the formula

$$
\frac{1}{2}\left\{\sigma_{n}^{\alpha}\left(\theta_{0}+\beta_{n}\right)-\sigma_{n}^{\alpha}\left(\theta_{0}-\beta_{n}\right)\right\}=-\frac{\sin n \beta_{n}}{A_{n}^{\alpha}} \sum_{j=0}^{\alpha} \tilde{s}_{n-j}^{j}\left(\theta_{0}\right) A_{j}^{\alpha-j}+o(1)
$$

analogous to the formula for $P_{n}+Q_{n}$ in $\$ 4.4$ (cf. (4.4.9)). Hence, making $n$ tend to $+\infty$ and using (4.8.7) we obtain

$$
-\underset{n \rightarrow \infty}{\liminf } \frac{1}{A_{n}^{\alpha}} \sum_{j=0}^{\alpha} \tilde{s}_{n-j}^{j}\left(\theta_{0}\right) A_{j}^{\alpha-j} \leqq \frac{1}{2}\left\{\sigma^{\alpha}\left(\theta_{0}\right)-\sigma_{\alpha}\left(\theta_{0}\right)\right\} .
$$

Simultaneously we consider the formula for $\tilde{\sigma}_{n}^{\alpha}\left(\theta_{0}\right)-\tilde{\sigma}_{n}^{\alpha+1}\left(\theta_{0}\right)$, which is analogous to (4.5.1), with $C_{\nu}\left(\theta_{0}\right)$ replaced by $\tilde{C}_{\nu}\left(\theta_{0}\right)$. It follows that

$$
\tilde{\sigma}_{n}^{\alpha}\left(\theta_{0}\right)-\tilde{\sigma}_{n}^{\alpha+1}\left(\theta_{0}\right)=\frac{n}{n+\alpha+1} \frac{1}{A_{n}^{\alpha}} \sum_{j=0}^{\alpha} \tilde{s}_{n-j}^{j}\left(\theta_{0}\right) A_{j}^{\alpha-j}+o(1)
$$

(cf. (4.5.3)). From this and (4.8.9) we get

$$
-\tilde{\sigma}_{\alpha}\left(\theta_{0}\right) \leqq \frac{1}{2}\left\{\sigma^{\alpha}\left(\theta_{0}\right)-\sigma_{\alpha}\left(\theta_{0}\right)\right\} .
$$

If $\tilde{s}\left(\theta_{0}\right) \neq 0$, this formula takes the form 


$$
\tilde{\sigma}_{\alpha}\left(\theta_{0}\right)-\tilde{s}\left(\theta_{0}\right) \geqq-\frac{1}{2}\left\{\sigma^{\alpha}\left(\theta_{0}\right)-\sigma_{\alpha}\left(\theta_{0}\right)\right\} .
$$

Applying this inequality, which is true for almost every point $\theta_{0}$ of $E$, to the series (4.8.3) multiplied by -1 , we get the inequality,

$$
\tilde{\sigma}^{\alpha}\left(\theta_{0}\right)-s\left(\theta_{0}\right) \leqq \frac{1}{2}\left\{\sigma^{\alpha}\left(\theta_{0}\right)-\sigma_{\alpha}\left(\theta_{0}\right)\right\} .
$$

The last two inequalities show that the series (4.8.3) is finite at the point $\theta_{0}$, and so almost everywhere in $E$.

Subtracting the last two inequalities we get the inequality

$$
\tilde{\sigma}^{\alpha}\left(\theta_{0}\right)-\tilde{\sigma}_{\alpha}\left(\theta_{0}\right) \leqq \sigma^{\alpha}\left(\theta_{0}\right)-\sigma_{\alpha}\left(\theta_{0}\right),
$$

true almost everywhere in $E$. The inequality opposite to this is also true, for the series (4.8.1) is summable $(C, \alpha+1)$ almost everywhere in $E$ (Lemma $M$ ), so that under the hypotheses of Lemma $O$ the series (4.8.1) and (4.8.3) play symmetric parts. (In particular, (4.8.5) is a consequence of the formula (4.4.4) in Lemma N.) It follows that (4.8.6) is true at the point $\theta_{0}$, and so almost everywhere in $E$. This completes the proof of Lemma $\mathrm{O}$ in the case of integral $\alpha$.

4.9. In the case of fractional $\alpha>-1$, the argument is identical with that of $\$ 4.6$, provided we assume that the series (4.8.3) is not only summable $(C, \alpha+1)$, but summable $(C, \beta)$, where $\beta=[\alpha]+1$, in $E$ (or almost everywhere in $E$ ).

It is therefore sufficient to show that if $\alpha$ is fractional the hypotheses of Lemma $\mathrm{O}$ imply that the series $(4.8 .3)$ is summable $(C, \beta)$ almost everywhere in $E$. First of all it may be remarked that if the series $(4.8 .1)$ is finite $(C, \alpha)$ and the series (4.8.3) finite $(C, \beta)$ almost everywhere in $E$, then the series (4.8.3) is also finite $(C, \alpha)$ almost everywhere in $E$ (the proof is substantially the same as the proof of the fact that if the series (4.8.1) is finite $(C, \alpha)$ and the series (4.8.3) summable $(C, \beta)$ in $E$, then the latter series is finite $(C, \alpha)$ almost everywhere in $E)$. On the other hand, the conditions of Lemma $O$ imply that the series (4.8.1) is finite $(C, \beta)$ and the series (4.8.3) summable $(C, \beta+1)$ in $E$, so that, using the lemma in the case of integral $\alpha$, we see that the series (4.8.3) is finite $(C, \beta)$, and so also finite $(C, \alpha)$, almost everywhere in $E$. Being finite $(C, \alpha)$ and summable $(C, \alpha+1)$ almost everywhere in $E$, the series (4.8.3) is summable $(C, \beta)$ almost everywhere in $E$ (Lemma $\mathrm{M}$ ). This completes the proof of Lemma $\mathrm{O}$ in the case of fractional $\alpha$.

4.10. Theorems 1 and 2 may now be proved in a few lines. Let us start with Theorem 1.

If the series (1.1.1) is summable $A$, and if $\sigma_{\alpha}(\theta)>-\infty$ at every point of a set $E$, then that series is summable $(C, \alpha+1)$ in $E$ (Lemma I). The conjugate series (1.1.2) is then summable $(C, \alpha+1)$ almost everywhere in $E$ (Lemma $M$ ). Theorem 1 is then a consequence of Lemmas $\mathrm{N}$ and $\mathrm{O}$.

In order to prove Theorem 2 , it is sufficient to show that under the hy- 
potheses of that theorem the series (1.1.1) is summable $A$ almost everywhere in $E$, for then Theorem 2 will follow from Theorem 1 .

From the hypotheses of Theorem 2 it follows that the series (1.1.1) is finite $\left(C^{*}, \alpha\right)$ almost everywhere in $E$ (Lemma $\mathrm{K}$ ), and so finite $A^{*}$ almost everywhere in $E$ (Lemma $F_{1}$ ). From Lemma $L$ it follows that the series (1.1.1). is summable $A$ almost everywhere in $E$.

This completes the proofs of Theorems 1 and 2 .

\section{PART II}

\section{The CASE OF POWER SERIES}

5.1. $\mathrm{By} \mathrm{K}^{0}(\zeta ; r)$ we shall denote the interior of the circle $\mathrm{K}(\zeta ; r)$, that is the set of points $z$ such that $|z-\zeta|<r$. Similarly, by $\mathrm{A}^{0}(\zeta ; r, R)$ we shall denote the interior of the annulus $\mathrm{A}(\zeta ; r, R)$.

Let us suppose that the power series

$$
\sum_{\nu=0}^{\infty} c_{\nu} e^{i \nu \theta}
$$

is summable $(C, \alpha+1)$ at a point $\theta$ to sum $t(\theta)$. Let $\tau_{n}^{\alpha}(\theta)$ denote the $\alpha$ th Cesàro means of the series (5.1.1). It will be convenient to consider the set of the limit points of the sequence of numbers

$$
\tau_{n}^{\alpha}(\theta)-t(\theta), \quad n=0,1,2, \cdots .
$$

This set of limit points we shall denote by $L_{1}^{\alpha}(\theta)$. The set $L_{1}^{\alpha}(\theta)$ is obtained from $\mathrm{L}_{\alpha}(\theta)$ by translating the latter by $-t(\theta)$.

The proof of Theorem 3 will be based on the following lemma.

Lemma P. Suppose that the series (5.1.1) is summable $(C, \alpha+1)$ (where $\alpha>-1)$ for every $\theta$ of a set $E$ of positive measure, and that for every $\theta$ of $E$ the set

$$
\mathrm{L}_{1}^{\alpha}(\theta)
$$

does not contain any point of a fixed circle $\mathrm{K}^{0}(\zeta ; r)$, with $|\zeta| \geqq r$. Then, for almost every $\theta$ of $E$, the set $\mathrm{L}_{1}^{\alpha}(\theta)$ does not contain any point of the annulus $\mathrm{A}^{0}(0 ;|\zeta|-r,|\zeta|+r)$.

Proof. Let $\left\{\delta_{n}(\theta)\right\}$ denote the sequence of functions defined on the set $E$ by the equation

$$
\delta_{n}(\theta)=\max \left(0, \delta_{n}^{\prime}(\theta)\right), \quad \text { where } r-\delta_{n}^{\prime}(\theta)=\inf _{\nu \geqq n}\left|\tau_{\nu}^{\alpha}(\theta)-t(\theta)-\zeta\right|\left({ }^{23}\right) .
$$

The functions $\delta_{n}(\theta)$ are measurable on $E$. They form a sequence decreasing monotonically to 0 . For a fixed $\theta$ and for $\nu \geqq n$, the numbers $\tau_{\nu}^{\alpha}(\theta)-t(\theta)$ lie outside the circle $\mathrm{K}^{0}\left(\zeta ; r-\delta_{n}(\theta)\right)$.

(23) By $\inf _{p} \geqq_{n} c_{p}$ we mean the largest lower bound of the sequence $c_{n}, c_{n+1}, \cdots$. 
Let $G$ denote any subset of $E$ on which the functions $\delta_{n}(\theta)$ tend uniformly to 0 and such that the function $t(\theta)$ is continuous on $G$. Let $\theta_{0}$ denote any point of density of $G$ belonging to $G$. Lemma $\mathrm{P}$ will have been proved when we have shown that the set $L_{1}^{\alpha}\left(\theta_{0}\right)$ contains no point of the annulus $\mathrm{A}^{0}(0 ;|\zeta|-r$, $|\zeta|+r)$. Without loss of generality we may assume that

$$
t\left(\theta_{0}\right)=0 \text {. }
$$

Let $\lambda$ be any real number and let $\left\{\beta_{n}\right\}$ denote a sequence of numbers such that

$$
n \beta_{n} \rightarrow \lambda, \quad \theta_{0}+\beta_{n} \in G \text { for } n=0,1,2, \cdots .
$$

By $t_{n}^{k}(\theta)$ we shall denote the $k$ th Cesàro sums of the series (5.1.1). On setting

$$
\begin{aligned}
C_{\nu}(\theta) & =c_{\nu} e^{i \nu \theta} & \text { for } \nu \geqq 0, \\
\mu_{\nu}=\mu_{\nu}^{(n)} & =A_{n-\nu}^{\alpha} e^{i \nu \beta_{n}} & \text { for } 0 \leqq \nu \leqq n,
\end{aligned}
$$

we get the formula

$$
\tau_{n}^{\alpha}\left(\theta_{0}+\beta_{n}\right)=\frac{1}{A_{n}^{\alpha}} \sum_{\nu=0}^{n} A_{n-\nu}^{\alpha} C_{\nu}\left(\theta_{0}\right) e^{i \nu \beta_{n}}=\frac{1}{A_{n}^{\alpha}} \sum_{\nu=0}^{n} C_{\nu}\left(\theta_{0}\right) \mu_{\nu} .
$$

This formula is entirely analogous to the formula (4.4.7). Since for our purposes the numbers $e^{i \nu \beta_{n}}$ have the same properties as the numbers $\cos \nu \beta_{n}$ (namely

$$
\left.\Delta^{k} e^{i \nu \beta_{n}}=O\left(\left|\beta_{n}\right|^{k}\right) \quad \text { for } k=0,1,2, \cdots\right),
$$

the argument which led from (4.4.7) to (4.4.9) gives in the case of integral $\alpha$ the formula

$$
\tau_{n}^{\alpha}\left(\theta_{0}+\beta_{n}\right)=\frac{e^{i n \beta_{n}}}{A_{n}^{\alpha}} \sum_{j=0}^{\alpha} t_{n-j}^{j}\left(\theta_{0}\right) A_{j}^{\alpha-j}+o(1) .
$$

Similarly, starting with the formula

$$
\tau_{n}^{\alpha}\left(\theta_{0}\right)-\tau_{n}^{\alpha+1}\left(\theta_{0}\right)=\frac{1}{n+\alpha+1} \frac{1}{A_{n}^{\alpha}} \sum_{\nu=0}^{n} \nu A_{n-\nu}^{\alpha} C_{\nu}\left(\theta_{0}\right),
$$

analogous to (4.5.1), we get the formula

$$
\tau_{n}^{\alpha}\left(\theta_{0}\right)-\tau_{n}^{\alpha+1}\left(\theta_{0}\right)=\frac{n}{n+\alpha+1} \frac{1}{A_{n}^{\alpha}} \sum_{j=0}^{\alpha} t_{n-j}^{j}\left(\theta_{0}\right) A_{j}^{\alpha-j}+o(1)
$$

corresponding to (4.5.3).

From (5.1.2), (5.1.3) and (5.1.4) it follows that

$$
\tau_{n}^{\alpha}\left(\theta_{0}+\beta_{n}\right)=e^{i n \beta_{n}} \frac{n+\alpha+1}{n} \tau_{n}^{\alpha}\left(\theta_{0}\right)+o(1) .
$$


Since $\tau_{n}^{\alpha+1}\left(\theta_{0}\right) \rightarrow 0$ (cf. (5.1.2)), we have $t_{n}^{\alpha+1}\left(\theta_{0}\right)=o\left(n^{\alpha+1}\right)$, so that

$$
t_{n}^{\alpha}\left(\theta_{0}\right)=t_{n}^{\alpha+1}\left(\theta_{0}\right)-t_{n-1}^{\alpha+1}\left(\theta_{0}\right)=o\left(n^{\alpha+1}\right) .
$$

Hence

$$
\tau_{n}^{\alpha}\left(\theta_{0}\right)=o(n) .
$$

It follows that the factor $(n+\alpha+1) / n$ on the right-hand side of (5.1.5) may be suppressed, and the formula may be rewritten in the form

$$
e^{-i n \beta_{n} \alpha} \tau_{n}^{\alpha}\left(\theta_{0}+\beta_{n}\right)=\tau_{n}^{\alpha}\left(\theta_{0}\right)+o(1) .
$$

This formula was obtained under the condition (5.1.2). The general case may be reduced to this special one by subtracting $t\left(\theta_{0}\right)$ from the constant term of the series (5.1.1). The formula (5.1.6) then takes the form

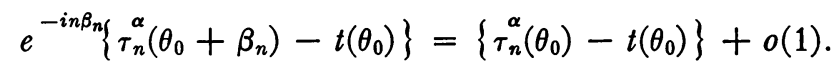

Since however the function $t(\theta)$ is by hypothesis continuous at the point $\theta_{0}$ with respect to the set $G$, so that

we get

$$
t\left(\theta_{0}\right)-t\left(\theta_{0}+\beta_{n}\right) \rightarrow 0,
$$

$$
e^{-i n \beta_{n}}\left\{\tau_{n}^{\alpha}\left(\theta_{0}+\beta_{n}\right)-t\left(\theta_{0}+\beta_{n}\right)\right\}=\left\{\tau_{n}^{\alpha}\left(\theta_{0}\right)-t\left(\theta_{0}\right)\right\}+o(1) .
$$

The formula (5.1.5), and so also the formula (5.1.7), holds in the case of fractional $\alpha>-1$, for then instead of (5.1.3) we have

$$
\tau_{n}^{\alpha}\left(\theta_{0}+\beta_{n}\right)=\frac{e^{i n \beta_{n}}}{A_{n}^{\alpha}}\left\{\sum_{\nu=0}^{n-\beta-1} t_{\nu}^{\beta}\left(\theta_{0}\right) A_{n-\nu}^{\alpha-\beta-1}+\sum_{j=0}^{\beta} t_{n-j}^{j}\left(\theta_{0}\right) A_{j}^{\alpha-j}\right\}+o(1)
$$

(cf. (4.6.2)), where $\beta=[\alpha]+1$, and instead of (5.1.4)

$$
\begin{aligned}
& \tau_{n}^{\alpha}\left(\theta_{0}\right)-\tau_{n}^{\alpha+1}\left(\theta_{0}\right) \\
& =\frac{n}{n+\alpha+1} \frac{1}{A_{n}^{\alpha}}\left\{\sum_{\nu=0}^{n-\beta-1} t_{\nu}^{\beta}\left(\theta_{0}\right) A_{n-\nu}^{\alpha-\beta-1}+\sum_{j=0}^{\beta} t_{n-j}^{j}\left(\theta_{0}\right) A_{j}^{\alpha-j}\right\}+o(1)
\end{aligned}
$$

(cf. (4.6.4))

By hypothesis, the points $\theta_{0}+\beta_{n}$ belong to $G$, so that the number $\tau_{n}^{\alpha}\left(\theta_{0}+\beta_{n}\right)-t\left(\theta_{0}+\beta_{n}\right)$ lies outside the circle $\mathrm{K}^{0}\left(\zeta ; r-\delta_{n}\right)$, where

$$
\delta_{n}=\sup \delta_{n}(\theta)\left({ }^{24}\right)
$$

for $\theta \in G$.

It follows that the left-hand side of the equation (5.1.7) does not belong to the circle $\mathrm{K}^{0}\left(\zeta e^{-i n \beta_{n}} ; r-\delta_{n}\right)$. Since $n \beta_{n} \rightarrow \lambda$ and $\delta_{n} \rightarrow 0$, the set $\mathrm{L}_{1}^{\alpha}\left(\theta_{0}\right)$ lies outside

(24) In other words, $\delta_{n}$ is the least upper bound of the sequence $\left\{\delta_{n}\right\}$ on $G$. 
the circle $\mathrm{K}^{0}\left(\zeta e^{-i \lambda} ; r\right)$. It is now sufficient to observe that $\lambda$ is an arbitrary real number, so that the set $\mathrm{L}_{1}^{\alpha}\left(\theta_{0}\right)$ lies outside the annulus $\mathrm{A}^{0}(0 ;|\zeta|-r,|\zeta|+r)$.

This completes the proof of Lemma $P$.

5.2. It is now easy to prove Theorem 3 .

Let $Z$ denote the set of points $\theta$ belonging to $E$ and such that the corresponding set $L_{1}^{\alpha}(\theta)$ is not of circular structure. The sets $L_{1}^{\alpha}(\theta)$ are closed. For every $\theta$ of $Z$ there is then a circle

$$
\mathrm{K}^{0}(\zeta ; r)
$$

with $|\zeta| \geqq r$

such that

(i) $\mathrm{L}_{1}^{\alpha}(\theta)$ contains no point of $\mathrm{K}^{0}(\zeta ; r)$;

(ii) $\mathrm{L}_{1}^{\alpha}(\theta)$ contains points of the annulus $\mathrm{A}^{0}(0 ;|\zeta|-r,|\zeta|+r)$.

We may suppose that the circle (5.2.1) is rational, that is, the three numbers $\xi, \eta, r$, where $\xi+i \eta=\zeta$, are rational.

Let $Z_{\zeta, r}$ denote the set of points $\theta$ of $E$ satisfying conditions (i) and (ii). By Lemma $P$, every set $Z_{\zeta, r}$ is of measure zero. Since

$$
Z=\sum Z_{\zeta, r}
$$

where it is sufficient to extend summation over all rational circles $\mathrm{K}^{0}(\zeta ; r)$, the measure of $Z$ is equal to 0 .

This completes the proof of Theorem 3 .

5.3. We now pass to the proof of Theorem 4. The theorem being obvious for $\alpha=0$, we may assume that $\alpha>0$. We have already observed in $\$ 1.4$ that it is sufficient to prove the relation (1.4.2).

From the formula

we deduce that

$$
A_{n}^{\alpha}=\frac{n+\alpha}{n} A_{n-1}^{\alpha}
$$

$$
\begin{aligned}
\tau_{n}^{\alpha}(\theta)-\tau_{n-1}^{\alpha}(\theta) & =c_{n} e^{i n \theta}+\sum_{\nu=0}^{n}\left(\frac{A_{n-\nu}^{\alpha}}{A_{n}^{\alpha}}-\frac{A_{n-\nu-1}^{\alpha}}{A_{n-1}^{\alpha}}\right) c_{\nu} e^{i \nu \theta} \\
& =c_{n} e^{i n \theta}+\sum_{\nu=0}^{n-1} \frac{A_{n-\nu}^{\alpha-1}}{n A_{n}^{\alpha}} \nu c_{\nu} e^{i \nu \theta}
\end{aligned}
$$

It is therefore enough to show that the expression

$$
\sum_{\nu=0}^{n-1} \frac{A_{n-\nu}^{\alpha-1}}{n A_{n}^{\alpha}} \nu\left|c_{\nu}\right|=\sum_{\nu=0}^{[n / 2]}+\sum_{\nu=[n / 2]+1}^{n}=S_{1}+S_{2}, .
$$

say, tends to 0 . Now

$$
S_{1}=\frac{O\left(n^{\alpha-1}\right)}{n A_{n}^{\alpha}} \sum_{\nu=0}^{[n / 2]} \nu\left|c_{\nu}\right|=O\left(n^{-2}\right) \sum_{\nu=0}^{n} \nu\left|c_{\nu}\right|=o(1),
$$




$$
S_{2}=\frac{o(n)}{n A_{n}^{\alpha}} \sum_{\nu=[n / 2]+1}^{n-1} A_{n-\nu}^{\alpha-1}=o\left(n^{-\alpha}\right) \sum_{\mu=0}^{n} A_{\mu}^{\alpha-1}=o\left(n^{-\alpha}\right) A_{n}^{\alpha}=o \text { (1). }
$$

This completes the proof $\left({ }^{25}\right)$.

\section{STRONG SUMMABILITY OF TRIGONOMETRIC SERIES}

6.1. We shall now prove a theorem on the strong summability of conjugate trigonometric series.

The series $\sum_{\nu=0}^{\infty} c_{\nu}$ is said to be strongly summable $(C, 1)$-or simply strongly summable - with index $q>0$, to sum $s$, if

$$
\frac{1}{n+1} \sum_{\nu=0}^{n}\left|s_{\nu}-s\right|^{q} \rightarrow 0 \quad \text { for } n \rightarrow \infty,
$$

where $s_{\nu}$ denote the partial sums of the series considered.

Theorem 5. If the series

$$
\frac{1}{2} a_{0}+\sum_{\nu=1}^{\infty}\left(a_{\nu} \cos \nu \theta+b_{\nu} \sin \nu \theta\right)
$$

is strongly summable, with index $q \geqq 1$, at every point of a set $E$, then the conjugate series

$$
\sum_{\nu=1}^{\infty}\left(a_{\nu} \sin \nu \theta-b_{\nu} \cos \nu \theta\right)
$$

is strongly summable, with index $q$, almost everywhere in $E$.

This theorem was stated by us in an earlier paper $\left({ }^{26}\right)$, with a sketch of proof. Since however the theorem has meanwhile been used in the proof of another result $\left({ }^{27}\right)$, we shall repeat here the proof of Theorem 7 in more detail.

Proof. Since $q \geqq 1$, the condition

$$
\frac{1}{n+1} \sum_{\nu=0}^{n}\left|s_{\nu}(\theta)-s(\theta)\right|^{q} \rightarrow 0
$$

implies

$$
\frac{1}{n+1} \sum_{\nu=0}^{n}\left|s_{\nu}(\theta)-s(\theta)\right| \rightarrow 0
$$

for $\theta \in E$. In particular, the series $(6.1 .1)$ is summable $(C, 1)$ in the set $E$. It

(25) A similar argument shows that the theorem holds in the case $-1<\alpha<0$, provided we replace the condition $c_{n} \rightarrow 0$ by $c_{n}=o\left(n^{\alpha}\right)$.

(26) See Marcinkiewicz and Zygmund [1].

(27) See Marcinkiewicz [1]. 
follows (Lemma $M$ ) that the series $(6.1 .2)$ is summable $(C, 1)$ at every point of a set $E_{1}$ contained in $E$ and of the same measure as $E$. Let $\tilde{s}(\theta)$ denote the $(C, 1)$ sum of the series (6.1.2), and let $G$ be a subset of $E_{1}$ such that

(i) the function $\tilde{s}(\theta)$ is continuous on $G$,

(ii) the relation (6.1.3) holds uniformly on $G$.

Theorem 5 will have been proved when we have shown that (6.1.2) is strongly summable at every point $\theta_{0}$ of $G$ which belongs to $G$ and is a point of density of $G$. Having fixed such a point $\theta_{0}$, we may assume without loss of generality that

$$
\tilde{\sigma}_{n}^{1}\left(\theta_{0}\right) \rightarrow 0
$$

Let $\left\{\alpha_{n}\right\}$ be any sequence of real numbers such that

$$
n \alpha_{n} \rightarrow \frac{1}{2} \pi,
$$

$$
\theta_{0} \pm \alpha_{n} \in G \text { for all } n>n_{0} .
$$

From the formula

$$
\begin{aligned}
s_{\nu}\left(\theta_{0}+\alpha_{n}\right)-s_{\nu}\left(\theta_{0}-\alpha_{n}\right)= & -\sum_{\mu=1}^{\nu}\left(a_{\mu} \sin \mu \theta_{0}-b_{\mu} \cos \mu \theta_{0}\right) \sin \mu \alpha_{n} \\
= & -\tilde{s}_{\nu}\left(\theta_{0}\right) \sin \nu \alpha_{n}-\tilde{s}_{\nu-1}^{1}\left(\theta_{0}\right) \Delta \sin (\nu-1) \alpha_{n} \\
& -\sum_{\mu=1}^{\nu-2} \tilde{s}_{\mu}^{1}\left(\theta_{0}\right) \Delta^{2} \sin \mu \alpha_{n}
\end{aligned}
$$

it is easy to see that

$$
s_{\nu}\left(\theta_{0}+\alpha_{n}\right)-s_{\nu}\left(\theta_{0}-\alpha_{n}\right)=-\tilde{s}_{\nu}\left(\theta_{0}\right) \sin \nu \alpha_{n}+\epsilon_{\nu, n} \quad \text { for } 0 \leqq \nu \leqq n,
$$

where $\epsilon_{\nu, n}$ tends to 0 when $\nu \rightarrow \infty, n \geqq \nu$.

Hence

$$
\begin{aligned}
\left|\tilde{s}_{\nu}\left(\theta_{0}\right)\right|^{q}\left|\sin \nu \alpha_{n}\right|^{q} \leqq & \left\{\left|s_{\nu}\left(\theta_{0}+\alpha_{n}\right)-s\left(\theta_{0}+\alpha_{n}\right)\right|+\left|s\left(\theta_{0}+\alpha_{n}\right)-s\left(\theta_{0}-\alpha_{n}\right)\right|\right. \\
& \left.+\left|s\left(\theta_{0}-\alpha_{n}\right)-s_{\nu}\left(\theta_{0}-\alpha_{n}\right)\right|+\left|\epsilon_{\nu, n}\right|\right\}^{q}
\end{aligned}
$$

and so

$$
\begin{aligned}
\frac{1}{n+1} \sum_{\nu=1}^{n}\left|\tilde{s}_{\nu}\left(\theta_{0}\right)\right|^{q}\left|\sin \nu \alpha_{n}\right|^{q} \leqq & \frac{4^{q-1}}{n+1} \sum_{\nu=1}^{n}\left|s_{\nu}\left(\theta_{0}+\alpha_{n}\right)-s\left(\theta_{0}+\alpha_{n}\right)\right|^{q} \\
& +\frac{4^{q-1}}{n+1} \sum_{\nu=1}^{n}\left|s\left(\theta_{0}+\alpha_{n}\right)-s\left(\theta_{0}-\alpha_{n}\right)\right|^{q} \\
& +\frac{4^{q-1}}{n+1} \sum_{\nu=1}^{n}\left|s\left(\theta_{0}-\alpha_{n}\right)-s_{\nu}\left(\theta_{0}-\alpha_{n}\right)\right|^{q} \\
& +\frac{4^{q-1}}{n+1} \sum_{\nu=1}^{n}\left|\epsilon_{\nu, n}\right|^{q} .
\end{aligned}
$$


The first and third terms on the right tend to 0 as $n \rightarrow \infty$, since the points $\theta_{0} \pm \alpha_{n}$ belong to the set $G$, on which the relation (6.1.3) holds uniformly. The second term on the right also tends to 0 , for it does not exceed the expression

$$
4^{q-1}\left|s\left(\theta_{0}+\alpha_{n}\right)-s\left(\theta_{0}-\alpha_{n}\right)\right|^{a} \rightarrow 0
$$

(cf. condition (i)). Since the last term on the right obviously tends to 0 , the left-hand side of (6.1.6) tends to 0 . On account of the first relation (6.1.5) we get

$$
\sum_{\nu=1}^{n}\left|\tilde{s}_{\nu}\left(\theta_{0}\right)\right| q^{q}=o\left(n^{q+1}\right)
$$

Let $S_{n}$ denote the sum on the left of (6.1.7). Then

$$
\begin{aligned}
\sum_{\nu=1}^{n}\left|\tilde{s}_{\nu}\left(\theta_{0}\right)\right|^{q} & =\sum_{\nu=1}^{n}\left(S_{\nu}-S_{\nu-1}\right) \nu^{-q}=\sum_{\nu=1}^{n-1} S_{\nu}\left(\nu^{-q}-(\nu+1)^{-q}\right)+S_{n} n^{-q} \\
& =\sum_{\nu=1}^{n-1} o\left(\nu^{q+1}\right) O\left(\nu^{-q-1}\right)+o\left(n^{q+1}\right) n^{-q}=o(n) .
\end{aligned}
$$

This completes the proof of Theorem $5\left({ }^{28}\right)$.

6.2. A slight modification of the above argument gives the following more general result.

THEOREM 6. Let $\phi(u), u \geqq 0$, be a convex strictly increasing function vanishing for $u=0$. There is an absolute constant $\lambda>0$ with the following property. If, for any series $(6.1 .1)$, the sequence $\phi\left(\left|s_{\nu}(\theta)-s(\theta)\right|\right)$ is summable $(C, 1)$ to 0 at every point $\theta$ of a set $E$, the sequence $\phi\left(\lambda\left|\tilde{s}_{\nu}(\theta)-\tilde{s}(\theta)\right|\right)$, where $\tilde{s}(\theta)$ is the $(C, 1)$ sum of $(6.1 .2)$, is summable $(C, 1)$ to 0 almost everywhere in $E$.

We may take for example $\lambda=1 / 10$.

\section{Generalization}

7.1. Theorem 3 deals with power series; Theorems 1 and 2 with real parts of power series. It is natural to inquire how far the results we have obtained hold for Dirichlet's series

$$
\sum_{\nu=1}^{\infty} c_{\nu} e^{-\lambda_{\nu} s}, \quad \lambda_{1}<\lambda_{2}<\cdots<\lambda_{n} \rightarrow \infty,
$$

or, more generally for the Stieltjes integral of the type

$$
\int_{0}^{\infty} e^{-\lambda s} d C(\lambda)
$$

(28) The above proof holds in the case $0<q<1$, if we add the condition that the series (6.1.2) is summable $(C, 1)$ in $E$. 
where $C(\lambda)$ is a function which is of bounded variation over any finite interval, and $s=u+i v$ is a complex variable.

It is not difficult to show that the results we proved for power series hold mutatis mutandis for Dirichlet's series and for the more general integrals (7.1.2). The extensions must of course use the theory of arithmetic means of the integrals of the form (7.1.2).

The arithmetic means $\tau_{w}^{\alpha}$ of order $\alpha$ for the integral (7.1.2) are defined by the formula

$$
\tau_{w}^{\alpha}(s)=\int_{0}^{w}\left(1-\frac{\lambda}{w}\right)^{\alpha} e^{-\lambda s} d C(\lambda)
$$

where $w$ is a continuous variable tending to $+\infty$, and $\alpha$ is any non-negative number. In the case of a Dirichlet series the expression (7.1.3) reduces to M. Riesz's typical means.

Let $A(\lambda)$ and $B(\lambda)$ denote respectively the real and the imaginary part of the function $C(\lambda)$, so that

$$
C(\lambda)=A(\lambda)-i B(\lambda) .
$$

If we suppose that the variable $s$ is purely imaginary,

$$
s=-i v,
$$

then the real and imaginary parts of the integral (7.1.2) are respectively equal to the integrals

$$
\begin{aligned}
& \int_{0}^{\infty} \cos \lambda v d A(\lambda)+\sin \lambda v d B(\lambda), \\
& \int_{0}^{\infty}-\cos \lambda v d B(\lambda)+\sin \lambda v d A(\lambda),
\end{aligned}
$$

and the second of these integrals will be spoken of as the conjugate to the first. If we denote the arithmetic means of order $\alpha$ for these two integrals by $\sigma_{w}^{\alpha}(v)$ and $\tilde{\sigma}_{w}^{\alpha}(v)$ respectively, then

$$
\begin{aligned}
& \sigma_{w}^{\alpha}(v)=\int_{0}^{w}\left(1-\frac{\lambda}{w}\right)^{\alpha}(\cos \lambda v d A(\lambda)+\sin \lambda v d B(\lambda)), \\
& \tilde{\sigma}_{w}^{\alpha}(\theta)=\int_{0}^{w}\left(1-\frac{\lambda}{w}\right)^{\alpha}(-\cos \lambda v d B(\lambda)+\sin \lambda v d A(\lambda)) .
\end{aligned}
$$

Let us write

$$
\begin{gathered}
\liminf _{w \rightarrow \infty} \sigma_{w}^{\alpha}(v)=\sigma_{\alpha}(v), \quad \underset{w \rightarrow \infty}{\lim \sup } \sigma_{w}^{\alpha}(v)=\sigma^{\alpha}(v), \\
\sigma^{\alpha}(v)-\sigma_{\alpha}(v)=\omega_{\alpha}(v),
\end{gathered}
$$


and let us define similarly the functions $\tilde{\sigma}_{\alpha}(v), \tilde{\sigma}^{\alpha}(v), \omega_{\alpha}(v)$ corresponding to the conjugate integral. Then the analogues of Theorems 1 and 2 for the integrals (7.1.4) may be stated as follows:

Theorem 1'. Suppose that the integral (7.1.4) is summable $A$ to sum $s(v)$, at every point of a set $E$ of positive measure, and that for an $\alpha \geqq 0$

$$
\sigma_{\alpha}(v)>-\infty, \quad v \in E .
$$

Then at almost every point of $E$ we have the relations

$$
\begin{aligned}
\sigma^{\alpha}(v) & <+\infty, \\
-\infty<\tilde{\sigma}_{\alpha}(v) & \leqq \tilde{\sigma}^{\alpha}(v)<+\infty, \\
\tilde{\omega}_{\alpha}(v) & =\omega_{\alpha}(v) .
\end{aligned}
$$

Moreover the integrals (7.1.4) and (7.1.5) are summable by the arithmetic means of order $>\alpha$ at almost every point of the set $E$ and

$$
\begin{aligned}
& s(v)=\frac{1}{2}\left\{\sigma_{\alpha}(v)+\sigma^{\alpha}(v)\right\}, \\
& \tilde{s}(v)=\frac{1}{2}\left\{\tilde{\sigma}_{\alpha}(v)+\tilde{\sigma}^{\alpha}(v)\right\},
\end{aligned}
$$

almost everywhere in $E$, where $\tilde{s}(v)$ denotes the value of the integral (7.1.5) by the method of arithmetic means of order $\alpha$.

THEOREM 2'. If the arithmetic means $\sigma_{w}^{\alpha}(v)$ of order $\alpha$ of the integral (7.1.4) are bounded at every point of a set $E$ of positive measure, then at almost every point of $E$ the integrals (7.1.4) and (7:1.5) are summable by the arithmetic means of order $>\alpha$, and we have the relations (7.1.7), (7.1.8), (7.1.9), (7.1.10).

In order to state the analogue of Theorem 3, let us suppose that the integral

$$
\int_{0}^{\infty} e^{i v \lambda} d C(\lambda)
$$

is at every point of a set $E$ summable by the method of arithmetic means to the value $t(v)$. By $L^{\alpha}(v)$ we shall denote the set of limit points of the expression

$$
\tau_{w}^{\alpha}(v)-t(v)\left({ }^{29}\right)
$$

(considered as a function of $w$ ), so that $\mathrm{L}^{\alpha}(v)$ is the set of the numbers

$$
\zeta=\lim _{n \rightarrow \infty}\left\{\tau_{w_{n}}^{\alpha}(v)-t(v)\right\}
$$

${ }^{(29)} \mathrm{By} \tau_{w}^{\alpha}(v)$ we mean the expression

$$
\int_{0}^{w}\left(1-\frac{\lambda}{w}\right)^{\alpha} e^{i \lambda \nu} d C(\lambda) .
$$


where $\left\{w_{n}\right\}$ is an arbitrary sequence of real numbers tending to infinity. Then we have the following.

THEOREM 3'. Suppose that at every point of a set E the integral (7.1.11) is summable by the method of arithmetic means of order $\alpha+1$, where $\alpha \geqq 0$, to the value $t(v)$. Then at almost every point of $E$ the set $\mathrm{L}^{\alpha}(v)$ is of circular structure with center $t(v)$.

There is no need to give here the proofs of Theorems $1^{\prime}, 2^{\prime}, 3^{\prime}$, since they are wholly analogous to the proofs of Theorems $1,2,3$. The theories of arithmetic means for ordinary series and for the integrals of the form (7.1.2) resemble so closely one another, and the technique of dealing with the arithmetic means of integrals is now so developed and so familiar (thanks mainly to the work of M. Riesz and G. H. Hardy $\left({ }^{30}\right)$ ) that the passage from series to integrals usually requires no new ideas.

A. F. Andersen

\section{REFERENCES}

1. Studier over Cesàro's Summabilitetsmetode, Copenhagen, Gjellerup, 1921.

G. H. HaRdy and J. E. LitTlewood

1. Contributions to the arithmetic theory of series, Proceedings of the London Mathematical Society, vol. 11 (1913), pp. 401-478.

G. H. HARDY AND M. RiEsZ

1. Dirichlet's Series, Cambridge Tracts, Cambridge, 1915.

J. KARAMATA

1. Über die Hardy-Littlewoodsche Umkehrungen des Stetigkeitssatzes, Mathematische Zeitschrift, vol. 32 (1930), pp. 319-320.

E. Kogbetliantz

1. Sommation des séries et intégrales divergentes, Mémorial des Sciences Mathématiques, vol. 51, pp. 1-75.

B. KUTTNER

1. A theorem on trigonometric series, Journal of the London Mathematical Society, vol. 10 (1935), pp. 131-140.

J. Marcinkiewicz

1. Sur la sommabilité forte des séries de Fourier, Journal of the London Mathematical Society, vol. 14 (1939), pp. 162-168.

J. Marcinkiewicz AND A. Zygmund

1. On the differentiability of functions and summability of trigonometrical series, Fundamenta Mathematicae, vol. 26 (1936), pp. 1-43.

A. Plessner

1. Über konjugierte trigonometrische Reihen, Comptes Rendus de l'Académie des Sciences de l'URSS, vol. 4 (1935), pp. 251-253.

2. Über das Verhalten analytischer Funktionen auf dem Rande des Definitionsbereiches, Journal für die reine und angewandte Mathematik, vol. 158 (1928), pp. 219-227.

I. PrivalofF

1. Sur une généralisation du théorème de Fatou, Récueil Mathématique de Moscou, vol. 31 (1923), pp. 232-235.

(30) See, for example, Hardy and Riesz [1]. 
W. RogosinkI

1. Über die Abschnitte trigonometrischer Reihen, Mathematische Annalen, vol. 95 (1925), pp. 110-134.

\section{A. ZYGMUND}

1. Trigonometrical Series, Monografie Matematyczne, vol. 5, Warsaw, 1935.

2. Einige Sätze aus der Theorie der divergenten Reihen, Bulletin de l'Académie Polonaise, 1928, pp. 309-331.

UNIVERSITY OF WILNO,

Wilno, Poland

Mt. Holyoke College, South Hadley, Mass. 